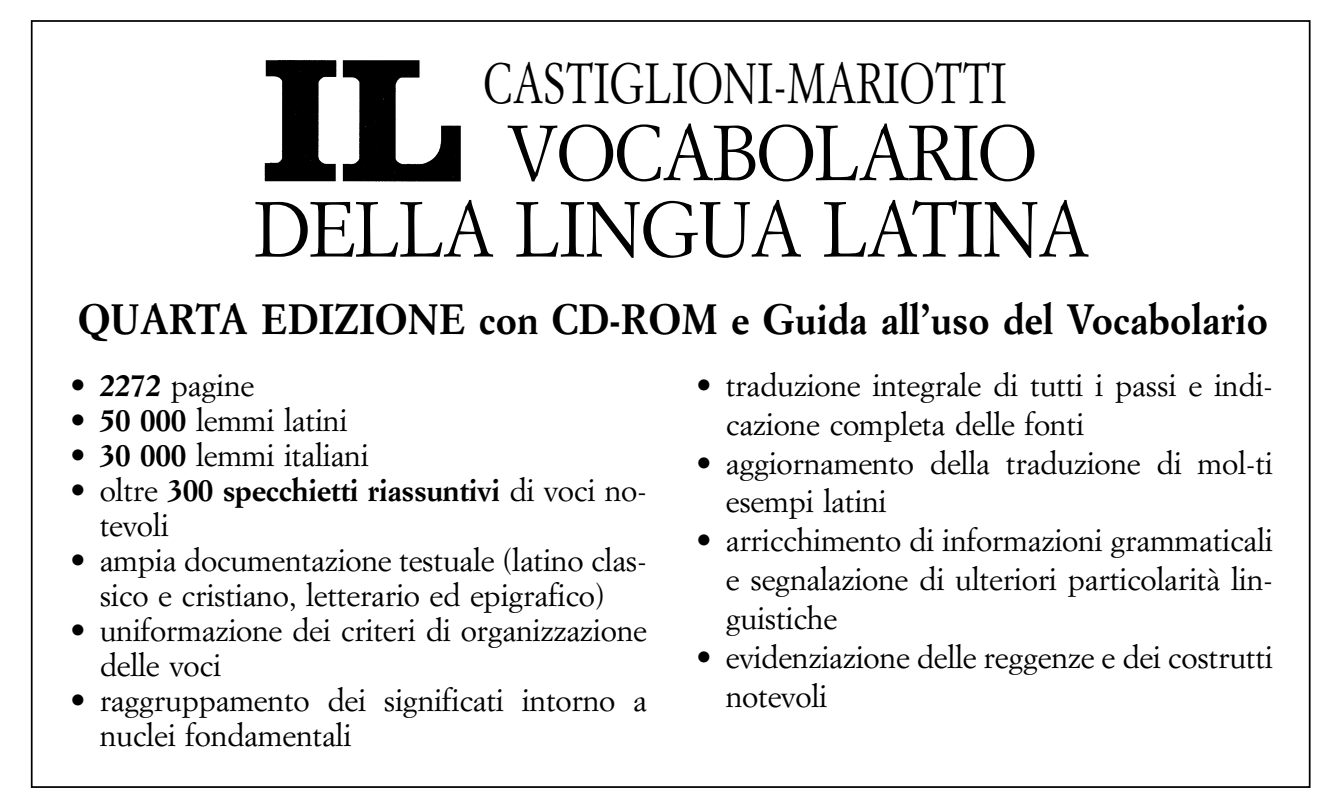
FRANCO MONTANARI VOCABOLARIO DELLA LINGUA GRECA
SECONDA EDIZIONE con CD-ROM e Guida all'uso del Vocabolario
- 2432 pagine
- 140000 lemmi tratti dalla letteratura ar- caica, classica, ellenistica, dai testi di età imperissical imperiale e tardo-antica, dalle opere dei prianze di papiri depigrafi
- circa 15000 vali sottoposti a esame si- - circa 15000 verbi sottoposti a esame si- stematico per componne 1 paradigmi in base alle fors realmente altestate neghi autori antichi
- 130 specchietti riassuntivi delle voci più complesse
- chiara strutturazione delle voci accompa- gnata da un fitta e articolata rete di esempi - costanti e puntuali citazioni dei luoghi e delle fonti
- evidenziazione delle reggenze e dei co- strutti sintattici
- esplicitazione di alcune abbreviazioni per evitare ambiguità

PAWAG (Poorly Attested Words in Ancient Greek) www.aristarchus.unige.it/pawag

Un repertorio on-line di termini della lingua greca scarsamente attestati a cura dell'Università di Genova in collaborazione con Loescher Editore.

Le versioni elettroniche di IL e GI sono disponibili in formato scaricabile all'indirizzo http://dizionariloescherit Tutte le informazioni sulle versioni elettroniche, on-line, per iPad e iPhone dei vocabolari sono reperibili alla pagina www.loescher.it/dizionari.

\section{MEDIACLASSICA}

www.loescher.it/mediaclassica

Un sito ricco di materiali e strumenti per lo studio e la didattica delle lingue classiche.

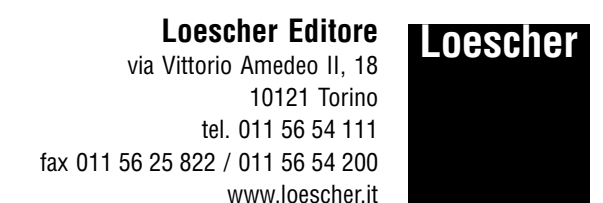

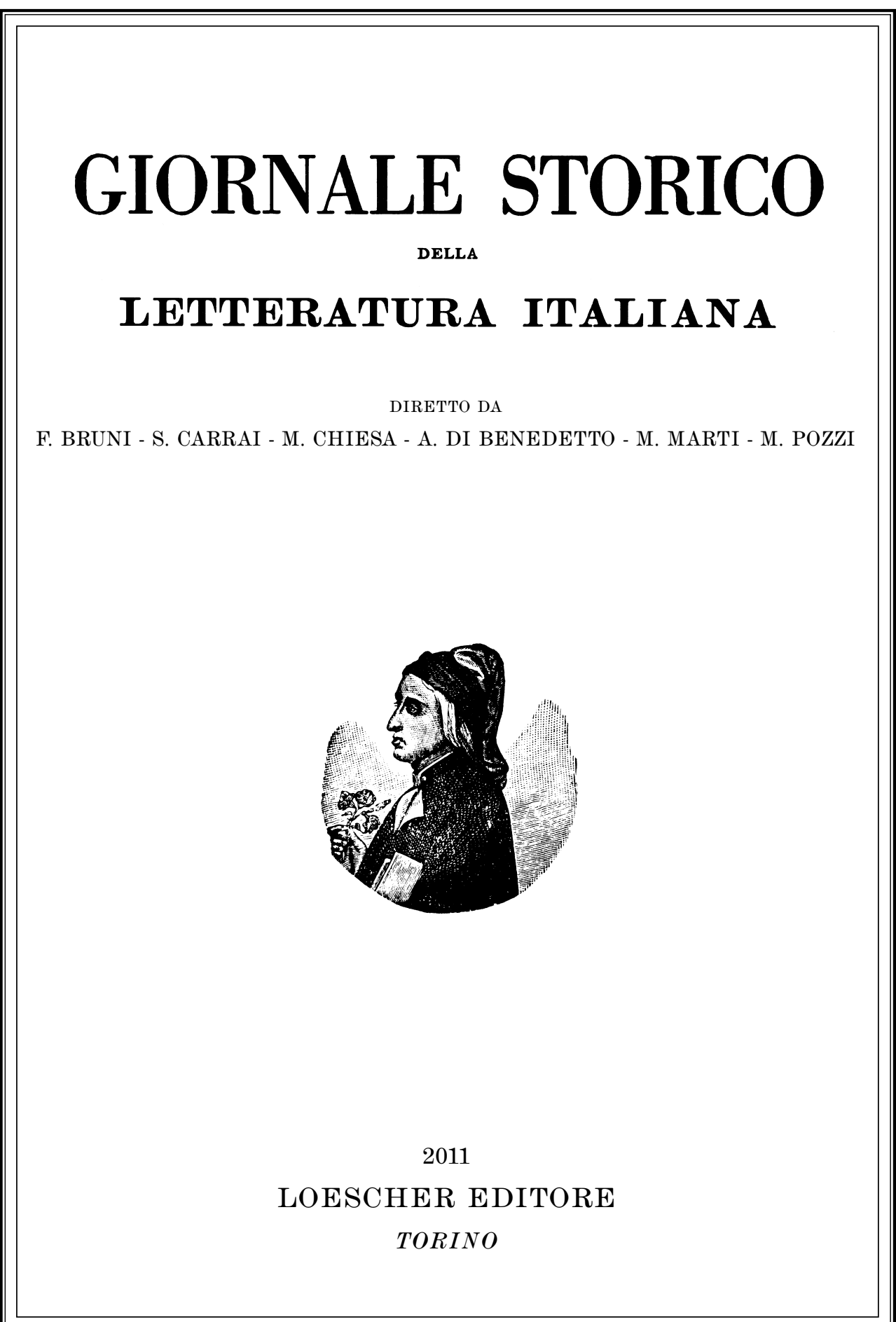

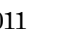


Zygmunt G. Baranski (Cambridge), Andrea Ciccarelli (Indiana University),

Jean-Louis Fournel (Paris VIII), Alfred Noe (Universität Wien),

FRANCISCO Rico (Universidad autónoma de Barcelona).

Maria Antonietta Terzoli (Universität Basel).

\section{SOMMARIO}

REDAZIONE

EnRico MatTioda (segretario), LOREnZo Bocca

Il «Giornale storico della letteratura italiana», fondato nel 1883 da Arturo Graf, Francesco Novati e Rodolfo Renier, e da allora pubblicato a Torino dalla Loescher, è punto di riferimento per gli studi di Italianistica.

E presente nelle maggiori biblioteche internazionali, ha ottenuto la qualifica ISI e si avvale della consulenza di lettori anonimi (peer review)

per la valutazione dei contributi proposti per la pubblicazione.

Contributi proposti per la pubblicazione e libri da recensire debbono essere inviati a: Giornale storico della letteratura italiana

Loescher Editore, via Vittorio Amedeo II, 18 - 10121 Torino e-mail: gsli@loescher.it

Coloro che desiderano sottoporre un contributo dovranno fare riferimento alle norme per la compilazione che sono scaricabili, in formato PDF, dal sito internet www.loescher.it/riviste

Nel medesimo sito sono consultabili i sommari dei fascicoli delle ultime annate, li abstrac deli articoli pubblicati, le informazioni su abbonamenti, ristampe anastatiche fascicoli arretrati e prezzi

Le annate del «Giornale storico della letteratura italiana» dal 1883 al 1995 sono inoltre consultabili on-line, previo abbonamento, nella banca dati Periodicals Archive Online

Modalità di pagamento 2011 (4 fascicoli annuali)

$$
\begin{aligned}
& € 86,00 \text { (Italia) }-€ 115.50 \text { (estero) } \\
& \text { Prezzo del singolo fascicolo: } € 29,00
\end{aligned}
$$

I versamenti vanno effettuati sul C.C.P. n. 96136007 , indirizzati a S.A.VE s.r.l ia Dell'Agricoltura 12 - 00065 Fiano Romano

Registrato al N. 571 del Registro Periodici del Tribunale di Torino
MARIA CRISTINA FIGORILLI, Orientarsi nelle «cose del mondo»: il Machiavelli "sentenzioso" di Anton Francesco Doni e Francesco Sansovino. . . . . . . STEFANO CARRAI, Parini e la tempesta (in margine a una recente edizione delle «Odi»).

VA R IET À

LUCIA CUOMO, Il motivo dei doni in «Provedi, saggio, ad esta visione» di Dante da Maiano.

MARCO FAINI, Notizie dalla biblioteca Queriniana: Sannazaro, Giovanni Francesco Caracciolo, il Pistoia, Pietro Aretino.

JOHNNY L. BERTOLIO, «La Torta» ovvero il primo idillio: Leopardi traduttore del «Moretum

\section{COMUNICAZIONI E APPUNTI}

RODOLFO SIGNORINI, Novità per la biografia di Giovanni Mario/Mario Filelfo. (Il testamento dell'umanista; Andrea Mantegna).

ENRICO GARAVELLI, Lusinghe e veleni nel carteggio Tommaseo-Scarabelli.

STEFANO CARRAI, Ammenda per una lettera sveviana ritrovata.

\section{BOLLETTINO BIBLIOGRAFICO}

Matteo Maria Boiardo, Timone - Orphei tragoedia, a cura di MariantonietTa AcoCella e Antonia Tissoni Benvenuti, (Matteo Favaretto), p. 446. - Antonio Galateo, Eremita, a cura di Sebastiano Valerio (Mario Marti), p. 455. - Brunoro Zampeschi, L'Innamorato, a cura di Armando Maggi, Chiara Montanari, MiChael Subialka, Sarah Christopher-Faggioli (Sergio Corsi), p. 457. - Corrado PEsTELLI, Carlo Antici e l'ideologia della Restaurazione in Italia (Angelo Fabrizi), p. 459. - Giovanna CoRdiBella, Hölderlin in Italia. La ricezione letteraria (A naldo Di Benedetto), p. 461. - MARIo MarTelli, Pascoli 1903-1904: tra rima sciolto (Giovanni Barberi Squarotti), p. 465.

a N N U N Z I , a cura di Lorenzo Bocca, Nó́mie Castagné, Mario Chiesa, Maria Luisa Doglio, Valentina Martino, Enrico Mattioda, Mario Pozzi. forte. - S. CRACOLICI. - B. DA FosSOMBRONE. - A.F. VERDE. - P. LARIVAILLE. - Ju stice et armes au XVIe siècle. - Gli dei a corte. - G. BarucCI. - E. DuRANTE-A. MARTELIOTTI - B. BALDI - Stagioni di un cantimbanco - I TEOTOCH ALBAZZI. G. Gassi - S. Baragetti - A. Fogazzaro-G. Giacosa - F. Chiesa - J. BerTRAND-A. PALAZZESCHI - «Filologia Veneta». 


\title{
LA TORTA OVVERO IL PRIMO IDILLIO: LEOPARDI TRADUTTORE DEL MORETUM
}

\author{
Carlo, Agnese e Becky \\ ridenti e fuggitivi
}

Spedii questo piccolissimo lavoro alla Sig. Compilatrice del C. delle D. perchè, quando le andasse a grado, gli dasse un luogo nel suo Giornale: onde ella prontamente lo inserì nei fogli diretti al pizzicagnolo. Io la prego a perdonarmi se nella lettera veramente molto breve, che le indirizzai, feci uso di espressioni poco convenienti, e dichiaro che le ritratto. Ivi sottometteva il mio lavoro al giudizio di lei (1).

Così nel giugno del 1816 il conte Giacomo Leopardi scriveva in una introduzione $\mathrm{Al}$ lettore alla seconda redazione della Torta. Il poeta aveva da poco concluso il volgarizzamento del poemetto pseudo-virgiliano Moretum e, di fronte alla sua mancata pubblicazione sul «Corriere delle Dame» (il "C. delle D." appunto), aveva pensato di diffonderne di propria iniziativa una bella copia nella cerchia dei famigliari (2). Soltanto nel dicembre dello stesso anno, il testo fu inviato all'editore Stella (3) che prontamente provvide a pubblicarlo sul numero de «Lo Spettatore Italiano» del 15 gennaio 1817. Si trattava di una versione nuova rispetto alle due precedenti (rimaste peraltro inedite) e sulla quale il traduttore sarebbe nuovamente intervenuto (nell'aprile del '17) per poi promuovere una ulteriore stampa realizzata nel 1822 «dalla tipografia Fratini di Recanati in occasione delle nozze di Camillo Antici con Marianna Ricci» (4); nonostante le correzioni, al tipografo fu comunque consegnato lo stesso testo già uscito su «Lo Spettatore», benché privato

(1) Dalla Nota introduttiva alla bella copia di a, in L. STEFAnI, "La Torta" di Giacomo Leopardi, in «Studi e problemi di critica testuale», V, 1972, p. 146.

(2) Stefani, "La Torta" di Giacomo Leopardi cit., p. 137.

(3) Cfr. Lettera a A.F. Stella del 27 dicembre 1816 (in G. LEOPARDI, Lettere, a cura di R. Damiani, Milano, A. Mondadori, 2006, p. 38). Leopardi invita l'editore a farne «l'uso che le piacerà» e lo Stella, poco prima dell'effettiva pubblicazione, risponderà: «vedrà il buon uso che ne ho fatto» (G. LEOPARDI, Epistolario, a cura di F. Brioschi e P. Landi, I, Torino, Bollati Boringhieri, 1998, p. 50).

(4) Stefani, "La Torta" di Giacomo Leopardi cit., p. 139. 
di introduzione e note, inadatte ad un opuscolo per nozze. Leopardi doveva tenere in qualche considerazione la propria traduzione se aveva previsto di inserirla nell'edizione bolognese dei Versi risalente al 1826 e affiancata dagli editori a quella delle Canzoni del '24. Come si evince dalla Prefazione secondo l'autografo (e da un ulteriore intervento correttorio successivo al 1822) (5), nella raccolta dei Versi dovevano trovarsi «tra le poesie originali la Guerra dei topi e delle rane e la Torta, perchè piuttosto imitazioni che traduzioni dal greco e dal latino» (6); in realtà, nella stampa si ritroverà soltanto la versione leopardiana della Batracomiomachia, e la Prefazione sarà conseguentemente riadattata.

Sul testo latino effettivamente utilizzato dal traduttore come "antigrafo" non c'è certezza, in mancanza di una esplicita dichiarazione da parte sua. Ė però stata appurata la presenza, nella biblioteca di casa Leopardi, di due volumi a cui Giacomo potrebbe aver fatto ricorso in modo contrastivo. Si tratta di edizioni di tutte le opere virgiliane (con tanto di apparato critico), compresi gli scritti oggi ritenuti di dubbia autenticità: la prima, veneziana, del 1562, la seconda, di Mannheim, del 1779: a quest'ultima il poeta accorda la propria preferenza negli unici due casi di lezione dubbia (7).

Un problema a parte è costituito dall'attribuzione del poemetto: dinnanzi a tale questione lo scrupolo filologico del giovane conte non rimase del tutto insensibile, ma nemmeno vi si applicò in maniera organica con gli strumenti, pur limitati, di cui disponeva (8). Nella nota (1) alla traduzione, il Moretum è definito un «vago poemetto [...] tra le cose dubbie di Virgilio» (9), anche se, presuppostane l'attribuzione virgiliana, la lingua del Moretum non è - Leo-

(5) Cfr. Stefani, "La Torta" di Giacomo Leopardi cit., p. 138.

(6) G. Leopardi, Poesie e Prose, I: Poesie, a cura di M.A. Rigoni, Milano, A. Mondadori, 1987, pp. 202-3.

(7) Cfr. Stefani, "La Torta" di Giacomo Leopardi cit., p. 142. Si tratta delle lezioni Vestaque/testaque (v. 51) e certisque/Nonisque (v. 78); in entrambi i casi, Leopardi opta per la seconda variante, attestata dall'edizione di Mannheim.

(8) Ovvio qui il rinvio a S. Timpanaro, La filologia di Giacomo Leopardi, RomaBari, Laterza, $1997^{3}$.

(9) Leopard, Poesie e Prose cit., I, p. 548. La suddetta nota è peraltro notevolmente debitrice nei confronti della Bibliotheca Latina del Fabricius dove, nel primo volume, si legge (si cita dalla quinta edizione pubblicata ad Amburgo nel 1721): «Moretum quod Virgilio ipsi adscribere aliqui cum Is. Vossio non dubitant, \& Parthenium Nicaenum poëtam Graecum in illo imitatum esse observant. Sub Svevii nomine laudat Salmasius in Solin. A. Septimio Severo post Scaligerum, Barthium aliosque tribuit Jo. Weitzius, qui notis illustravit Francof. 1642.8. ad calcem Copae. Graeca metaphrasis Jos. Scaligeri inter ejus Poëmata occurrit, ab auctore oblata Petro Ronsardo A. 1563» (pp. 223-4); di nuovo si parla del Moretum a p. 675 dove compare l'oscillazione Sereno/Severo quanto al cognomen del possibile autore. Nella sua nota Leopardi ha quasi letteralmente tradotto, dopo averli congiunti, i due passi del Fabricius. 
pardi lo sa bene - quella «del civilizzatissimo Virgilio, bensì [...] di uno pseudo-Virgilio rusticano e terrigno» (10). Quale autore è citato Svevio e, con maggiore convinzione anche per via dell'avallo «dello Scaligero, del Barthio, dello Weitzio [...] A. Settimio Sereno o Severo, poeta Falisco, vivuto come pare, al tempo de' Vespasiani» (11). A destare l'attenzione di Leopardi contribuì un ulteriore dettaglio che dotava il carme di un fascino particolare; un codice della Biblioteca Ambrosiana presentava (e presenta tuttora) in incipit la dicitura: «Parthenius (parlasi di Partenio di Nicea, poeta greco del secolo d'Augusto) Moretum scripsit in graeco, quem Virgilius imitatus est» (12). Tale glossa erudita, ancorché priva di un reale riscontro, era tuttavia cruciale per il Nostro, per la cui sensibilità il Moretum non solo rappresentava un idillio del secolo aureo della letteratura latina, ma incarnava in sé l'ideale stesso della classicità intesa come civiltà antica; il testo rappresentava in modo emblematico il travaso della letteratura e della civiltà greca nel mondo romano: «i latini non ebbero e non fecero altra opera che traspiantare di netto le scienze, arti, lettere greche nel loro terreno» (13). È forse per questo che, di fronte al Moretum, il filologo presto cede il posto al volgarizzatore e al poeta, che tuttavia, in forza della propria erudizione, mette in atto, del testo antico, una fruizione completa e non puramente strumentale (14). Quanto al metro, come già

(10) F. D'Intino in G. Leopardi, Poeti latini e greci, a cura di F. D'Intino, Roma, Salerno, 1999, p. XXXVI. Per un commento al testo latino del poemetto, tradotto - tra gli altri - da C. MARCHESI, Il "Moreto": carme attribuito a Virgilio, presentato e tradotto da C. M. in Idem, Scritti minori di filologia e letteratura, III, Firenze, Olschki, 1978, pp. 1311-8 e da R. HeInZE, Das Kräuterkäsgericht (Moretum) in Idem, Vom Geist des Römertums, a cura di E. Burck, Stuttgart, Teubner, 19603, pp. 40416, rinvio all'ottimo [P. Vergili Maronis] Moretum, a cura di A. Perutelli, Pisa, Giardini, 1983 (da integrare con Idem, Epilegomeni al Moretum, in «Materiali e discussioni per l'analisi dei testi classici», XXII, 1989, pp. 189-200) e, in seconda istanza, al quasi coevo The Ploughman's lunch. Moretum: a poem ascribed to Virgil, a cura di E.J. Kenney, Bristol, Bristol Classical Press, 1984; si veda infine anche Moretum, a cura di C. Laudani, Napoli, Loffredo, 2004, con aggiornatissima bibliografia.

(11) Leopard, Poesie e Prose cit., I, p. 548. Leopardi segue l'indicazione dello Scaligero (cfr. A. Perutelli, Moretum in Enciclopedia virgiliana, III, Roma, Istituto dell'Enciclopedia Italiana, 1987, p. 585).

(12) Leopard, Poesie e Prose cit., I, p. 548. Trattasi di «una notizia riportata in un codice umanistico» (Perutelli, Moretum cit., p. 586); cfr. R. SABbadini, Partenio $e$ il 'Moretum', in «Rivista di filologia e di istruzione classica», XXXI, 1903, p. 472 e Idem, Ancora Partenio e il 'Moretum', in «Rivista di filologia e di istruzione classica», XLIII, 1915, pp. 80-81, che spiega come Parthenias, con cui era appellato Virgilio (lo attesta la Vita Donatiana 11), potesse essere facilmente frainteso con Parthenius per via della notizia riportata da MACR. Sat. 5, 17, 18, per cui Partenio di Nicea fu maestro di Virgilio.

(13) Zib. 747.

(14) «Accanto al filologo in Leopardi c'è sempre il poeta» (E. PASQUinI, Leopardi fra traduzione e citazione: due trafile distinte, in La critica del testo. Problemi di metodo ed esperienze di lavoro. Atti del Convegno di Lecce - 22-26 ottobre 1984, Roma, 
nel caso della Guerra dei topi e delle rane, per evitare di «comporre piuttosto che tradurre» e di impiegare «rime stiracchiate [...] nemiche capitali della bellezza della poesia, e del piacere dei lettori» (gli effetti collaterali dell'ottava rima), Leopardi «scelse le sestine endecasillabe, dei vantaggi delle quali, dopo l'uso felicissimo che hanno fatto di loro parecchi poeti [...], non può più dubitarsi» (15).

L'esercizio di traduzione, per il Leopardi di questi anni, aveva raggiunto il suo vertice e stava per lasciare il posto a una nuova, originale stagione poetica. Tra il 1815 e il '16 avevano visto la luce La Guerra dei topi e delle rane, gli idilli di Mosco, il Saggio di traduzione dell'Odissea, le Iscrizioni greche triopee, la Traduzione del libro secondo della Eneide e di una parte del terzo (16) - tutti lavori che avevano ottenuto giudizi buoni, almeno a dar retta allo Stella: «ho da tutte parti ricevuto elogj delle sue traduzioni» (17). È indubbio il ruolo di apprendistato poetico svolto da queste versioni, un vero e proprio tirocinio che diventava anche «un modo di vivere la poesia dei classici» (18) e si affiancava, traghettandola verso la fonte Castalia, all'inesausta attività filologica. Nelle pagine dello $Z i$ baldone e nelle lettere che risalgono a questi fecondi anni, il traduttore ritorna a più riprese sul proprio rapporto con i classici ed è quasi per contenere l'impeto che gli sorge nell'animo dopo la loro lettura che il volgarizzare diviene un'esigenza - e non necessariamente dai capolavori o, dei capolavori, dai passi più noti: «quando ho letto qualche Classico, la mia mente tumultua e si confonde. Allora prendo a tradurre il meglio, e quelle bellezze per necessità esaminate e rimenate a una a una, piglian posto nella mia mente e l'arricchiscono e mi lasciano in pace» (19). L'esercizio della traduzione, inizialmente addirittura confuso con la vocazione poetica, costituisce una sorta di rielaborazione dell'iniziale impressione di turbamento risvegliata in Giacomo dal contatto diretto con i testi classici, avvertiti come l'unica eredità di un mondo ormai perduto (20) e ricondotti, in particolare, alla materialità della loro essenza - il ver-

Salerno, 1985, p. 608; cfr. S. ORLANDO, Il pessimismo antico nel Leopardi traduttore in Studi in onore di Alberto Chiari, II, Brescia, Paideia, 1973, pp. 911-4 e A. PRETE, Le pagine di Leopardi sul tradurre, in «Testo a fronte», V, 1991, p. 134).

(15) LeOpard, Poesie e Prose cit., I, pp. $418-9$ (nel Discorso sopra la Batracomiomachia).

(16) Cfr. E. BigI, Il Leopardi traduttore dei classici (1814-1817), in questo «Giornale», CXLI (1964), pp. 191-204.

(17) Lettera di A.F. Stella del 27 novembre 1816 (in LEOPARDI, Epistolario cit., p. 36).

(18) R. Pellegrini, La traduzione letteraria nel pensiero del Leopardi, in «Lettere italiane», XXX, 1978, p. 170.

(19) Lettera a P. Giordani del 21 marzo 1817 (in LeOPARDI, Lettere cit., p. 49).

(20) Cfr. F. D'Intino in Leopard, Poeti latini e greci cit., pp. XI e XV. 
bo che si fa carne nella lingua, lo stile come eccezionale residuo dell'antichità (21).

Inoltre, «il 1816 è l'anno delle lettere alla "Biblioteca Italiana", con le quali egli esce dal suo isolamento culturale per entrare nel vivo della polemica classico-romantica; o meglio, per tentare di entrarvi, dal momento che l'Acerbi non pubblica né la prima né la seconda» (22); in esse il loro autore, non soltanto si pronunciava sugli aspetti del tradurre (23), sull'onda della polemica innescata dal celebre articolo di Madame de Staël "Sulla maniera e utilità delle traduzioni”, ma affermava con forza l'originalità di forma e di contenuto delle opere dell'antichità, le quali, prive di modelli di riferimento, sgorgavano direttamente dalla natura, attuandone una mimesi scevra di sovrastrutture ed estremamente aderente al dato di fatto: «il più grande di tutti i poeti è il più antico, il quale non ha avuto modelli» (24). In questo senso, la scelta di tradurre il Moretum è stata interpretata come «squisitamente ideologica» (25): Simulo, il protagonista del poemetto, appartiene ad un mondo anti$\mathrm{co}$, forse addirittura greco, in cui l'assidua frequentazione della natura da parte dell'uomo, l'attività nei campi capillarmente diffusa, l'assoluta mancanza di tecnologia segnavano ogni istante della vita quotidiana. L'anonimo lasciava intravedere al giovane Giacomo uno spaccato dell'antichità che la vocazione prevalentemente agricola di Recanati per molti aspetti rispecchiava (26). Non soltanto il Moretum apparteneva al «buon tempo» (27) sotto il profilo cronologico, quale portato storico dell'antichità, ma del "buon tempo" ritraeva

(21) «Nelle opere moderne lo stile è cosa piccolissima o niente, nelle antiche è grandissima parte o il tutto» scriverà, nel 1826, nel Preambolo del volgarizzatore che precede le Operette morali di Isocrate (G. LeOPard, Poesie e Prose, II: Prose, a cura di R. Damiani, Milano, A. Mondadori, 1988, p. 1074, ma si tratta di un concetto più volte ribadito nello Zibaldone). Cfr. M.A. RigonI, L'estetizzazione dell'antico in Idem, Il pensiero di Leopardi, Milano, Bompiani, 1997, pp. 41-42: «che cosa determina il "classicismo" di Leopardi, se non il fatto che il suo materialismo è innanzitutto una teoria dello stile? Se il mondo è materia, esso conta come fenomeno estetico [...]. La poesia non è dunque poesia se non in quanto è materiale».

(22) Stefani, "La Torta" di Giacomo Leopardi cit., p. 167.

(23) Su questo aspetto cfr. M. Coco, Leopardi e la traduzione poetica, in «Otto/Novecento», XII, 1988, 1, pp. 159-62.

(24) Dalla Lettera ai Sigg. Compilatori della Biblioteca Italiana in risposta a quella di Mad. la Baronessa di Staël Holstein ai medesimi (inviata da Recanati il 18 luglio 1816, in Leopard, Poesie e Prose cit., II, p. 437).

(25) Stefani, "La Torta" di Giacomo Leopardi cit., p. 168.

(26) Su questo aspetto di riecheggiamento, nell'attualità quotidiana del borgo recanatese, della tradizione letteraria classica e italiana, si vedano le illuminanti conclusioni di F. BRuni, Il canto della donna al telaio e il dialogo con l'assente: "A Silvia" di Leopardi, in questo «Giornale», CLXXXII (2005), pp. 1-41 (parzialmente già edito in "Vaghe stelle dell'Orsa...". L' "io" e il "tu" nella lirica italiana, a cura di F. Bruni, Venezia, Marsilio, 2005, pp. 241-63).

(27) Leopardi, Poesie e Prose cit., I, p. 548. 
proprio i fossili di arcaicità, che i contadini della schiatta di Simulo, loro malgrado, contribuivano a conservare. Certamente, per candida ammissione del diretto interessato, l'obiettivo primo delle traduzioni era quello di procacciarsi fama fra gli intellettuali, al punto che la versione del secondo libro dell'Eneide fu inviata contemporaneamente ad Angelo Mai, al Monti e al Giordani (28); stupisce dunque che Leopardi si sia voluto dedicare al Moretum, tanto più che aveva già declinato l'offerta, fattagli dallo Stella, di tradurre Apollonio: «se io fossi in grado di dare ad alcuno consigli, darei a Lei quello di voltare in bel verso toscano l'Apollonio Rodio» (29) aveva suggerito l'editore per via dell'assenza di lavori adeguati in proposito; tuttavia, il contino, quasi risentito, vi rinunciò per dedicarsi all'Odissea: «se si tratta di acquistar fama, certe imprese non mai tentate non sono le più proprie per questo effetto [...]. Così ella vede che il Monti è assai più famoso per l'Iliade che pel Persio» (30).

La questione del tradurre, ovvero dell'imitare senza alterare drasticamente l'originale, ma anche senza sconvolgere le peculiarità della propria lingua (31), era, agli occhi di Leopardi, di importanza capitale: su questa problematica premessa teorica il poeta ritorna più volte soprattutto nelle introduzioni ai propri lavori, oltre che nelle già citate lettere indirizzate alla «Biblioteca Italiana»; in esse, accanto alle definizioni squisitamente filologiche, si rinvengono osservazioni precise relativamente a traduzioni precedenti della stessa opera - quel che compare, seppur in forma scarna, nella nota iniziale apposta alla Torta. Un primo punto riguarda la legittimità del tradurre: questo consiste essenzialmente nel volgere in un'altra lingua, ma anche in un'altra cultura, civiltà, mentalità, un testo nato in un mondo diverso e lontano. I classici poi, i quali vichianamente rappresentano per Leopardi la fanciullezza dell'umanità, sono il prodotto di uno spirito non toccato da quell'afflato civilizzatore del «se-

(28) Le lettere furono inviate contemporaneamente da Recanati il 21 febbraio 1817. Cfr. P. Fasano, Come gli antichi Greci in Idem, L'entusiasmo della ragione. Il romantico e l'antico nell'esperienza leopardiana, Roma, Bulzoni, 1985, pp. 68-69: «ma le risposte non furono esattamente entusiasmanti».

(29) Lettera di A.F. Stella del 27 novembre 1816 (in LEOPARDI, Epistolario cit., p. 36).

(30) Lettera a A.F. Stella del 6 dicembre 1816 (in LEOPARDI, Lettere cit., p. 31).

(31) Cfr. Pellegrini, La traduzione letteraria cit., p. 181: in base alle riflessioni formulate da Leopardi, «il traduttore [...] non deve fagocitare l'originale, ma rispettarne la diversità culturale; d'altra parte, per il rispetto dovuto alla propria tradizione, non deve turbare l'individualità della propria lingua». Si veda inoltre N. BELLUCCI, "Difficoltà e impossibilità di ben tradurre". Teoria e pratica della traduzione nei pensieri dello Zibaldone, in Lo Zibaldone cento anni dopo. Composizione, edizioni, temi. Atti del X Convegno internazionale di studi leopardiani-Recanati-Portorecanati 14-19 settembre 1998 (a cura di R. Garbuglia), I, Firenze, Olschki, 2001, p. 50. 
col superbo e sciocco» (32); il ritorno alla naturalità ai moderni è tuttavia precluso tanto quanto lo è l'obiettivo di attingere ai testi antichi nella stessa maniera dei loro primi fruitori: perciò, per via di un crescente pessimismo che sembra investire perfino la possibilità stessa di far rinascere i classici, «la coscienza della impossibilità di ogni traduzione è legata strettamente alla coscienza dell'impossibilità di un ritorno allo stato di natura» (33). Si intuisce però una certa ambiguità nell'atteggiamento del giovane Giacomo rispetto all'arte del tradurre: ora infatti egli afferma con forza la necessità assoluta del rispetto dell'originale - lo si coglie a chiare lettere nella critica mossa al Caro traduttore dell'Eneide, contenuta nel discorso introduttivo alla versione della Titanomachia di Esiodo (1817):

Io trovo troppo vizioso il maggior pregio della traduzione del Caro. Il quale sta in quella scioltezza, o volete disinvoltura, che fa parere l'opera non traduzione, ma originale. E questa s'ha procacciata il Caro con usar parole e frasi al tutto proprie della lingua nostra, e modi non ignobilmente volgari, che danno all'opera un colore di semplicità vaghissima e di nobile famigliarità. [...] Ora s'egli è obbligo stretto del traduttore il conservare anche i minutissimi lineamenti del testo, l'averne tramutato il distintivo e la proprietà principale [scilicet il dire sempre grande, sempre magnifico, sempre segnalatamente nobile, sempre superiore a quello del comune degli uomini proprio dello stile di Virgilio], certo sarà gran peccato (34).

La stessa persona che criticava le traduzioni troppo libere, come, a suo giudizio, quella del Caro, qualche anno prima (nel 1815) si era vantata, nel Discorso sopra la Batracomiomachia, di aver dato al poemetto pseudo-omerico una patina nuova:

tradussi non letteralmente [...], ma pur tradussi, e fui ben lontano dal fare un nuovo poema [...]. Cercai d'investirmi dei pensieri del poeta greco, di rendermeli propri, e di dar così una traduzione che avesse qualche aspetto di opera originale, e non obbligasse il lettore a ricordarsi ad ogni tratto che il poema, che leggea, era stato scritto in greco molti secoli prima. Volli che le espressioni del mio autore, prima di passare dall'originale nelle mie carte, si fermassero alquanto nella mia mente, e conservando tutto il sapor greco, ricevessero l'andamento italiano, e fossero poste in versi non duri e in rime che potessero sembrare spontanee (35)

- quella stessa spontaneità modernizzante perseguita, sempre a livello rimico, nella traduzione del Moretum. Si tratta di una vera e propria contraddizione per la quale l'unica via d'uscita pare essere quella di appellarsi allo «sperimentalismo piuttosto incerto e passivo del giovanissimo poeta» (36); l'alternativa, forse più appetibile, consisterebbe nel giustificare queste oscillazioni alla luce dei testi re-

(32) G. LEOPARDI, La ginestra o il fiore del deserto, v. 53.

(33) Stefani, "La Torta" di Giacomo Leopardi cit., p. 171.

(34) G. LeOpardi, [Preambolo alla] Titanomachia di Esiodo, a cura di P. Mazzocchini, Roma, Salerno, 2005, $\$ \$ 28-33$, pp. 42-45.

(35) Leopardi, Poesie e Prose cit., I, p. 419.

(36) BIGI, Il Leopardi traduttore dei classici cit., p. 196. 
cati in italiano (37). In altre parole, l'apprezzamento per una maggiore o minore fedeltà all'originale segue, non precede la realizzazione concreta della traduzione: di fronte ad un colosso della classicità, un Omero o un Virgilio, Leopardi sente fortissima l'esigenza di un rispetto ossequioso del testo, che, nel volgarizzatore moderno, non ammette alterazioni di sorta; d'altra parte, i figli cadetti delle auree nozze, la Batracomiomachia o il Moretum, maggiormente risultavano passibili di infedeltà e innovazioni al punto da dare vita più a «imitazioni che [a] traduzioni» (38) - già lo avevano percepito gli editori bolognesi. Non si tratta dunque di un cambiamento di mentalità intervenuto a distanza di tempo, ma di un diverso atteggiamento dipendente dal testo da volgarizzare. È così che, nel caso del Moretum, Leopardi si sente molto più libero e meno vincolato, rispetto all'Eneide, quanto alla resa dell'originale: «la misura e la tematica dell'idillio sono molto più vicine alla sua ispirazione di quanto non sia la solennità virgiliana: perciò può essere traduttore e poeta insieme» (39). Ma sarà proprio la traduzione del secondo libro dell'Eneide, quasi parallela a quella del Moretum, a segnare non solo un punto di svolta nell'approccio leopardiano con i classici (letti non più attraverso le lenti della «pura e secca filologia») (40), ma una presa di coscienza sempre più netta del ruolo di un buon traduttore (41). L'italiano, del resto, si prestava moltissimo, più di tutte le

(37) Fasano, Come gli antichi Greci cit., pp. 61-63 fa notare come la continua oscillazione fra il perseguimento della fedeltà e la ricerca di originalità siano in realtà le due facce della stessa medaglia, le quali, se all'inizio sembrano, quasi meccanicamente, l'una l'alternativa dell'altra, finiscono per diventare complementari. Si vedano inoltre B. NACCI, Leopardi teorico della traduzione, in «MLN», CXIV, 1999, p. 64 ( «traspare, per niente in contraddizione con il quadro teorico, un pragmatismo dettato dall'esperienza e dal gusto») e BeLlucci, "Difficoltà e impossibilità di ben tradurre" cit., p. 42.

(38) LeOPARDI, Poesie e Prose cit., I, p. 203. Nella produzione di Leopardi, due componimenti di ispirazione simonidea (Dal greco di Simonide, già parzialmente inserito nelle Operette morali, e Dello stesso) saranno posti in appendice ai Canti quasi fossero libere interpretazioni (cfr. ORLANDO, Il pessimismo antico cit., pp. 926-37, dove si inquadrano i due frammenti nel pensiero del traduttore che ne desume spunti significativi per i propri Canti, e PASQUINI, Leopardi fra traduzione e citazione cit., pp. 607-13). L'imitazione, a metà strada tra la semplice traduzione e la produzione poetica originale, sarà invece applicata alle libere versioni da Archiloco e da alcuni autori della commedia di mezzo, nate parallelamente a quelle simonidee (cfr. LEOPARDI, Poesie e Prose cit., I, pp. 605-7). Il passaggio successivo consisterà nel vero e proprio "falso", di cui l'esempio più significativo è costituito dai Paralipomeni della Batracomiomachia (ma siamo ormai negli anni Trenta). Leopardi pare praticare, sul piano poetico, quella stessa dialettica tra traduzioni letterali, oratorie fedeli e oratorie libere carissima agli umanisti del '400; tale classificazione, non sempre di felice applicazione, risale a R. SABBADINI, Il metodo degli Umanisti, Firenze, Felice Le Monnier, 1922, pp. 23-25.

(39) StEFAnI, "La Torta" di Giacomo Leopardi cit., p. 172.

(40) Zib. 193 (29 luglio 1820).

(41) Cfr. M. MARTI, Leopardi, Giacomo in Enciclopedia virgiliana, III, Roma, Isti- 
lingue moderne, all'operazione di "travaso" sia in virtù della propria adattabilità «a ogni sorta di stili» (42) sia in virtù del proprio statuto "romanzo".

In questo percorso di progressiva acquisizione di un compito preciso, che apre la via al celebre «passaggio [...] dall'erudizione al bello non [...] subitaneo, ma gradato» (43), va collocata anche la composizione della Torta - alla luce di quanto detto volgarizzamento non soddisfa più. Anche (senz'altro non solo) grazie al Moretum l'impeto della vocazione poetica diventerà sempre più urgente fino a provocare una sorta di sovvertimento nel rapporto tra traduttore e scrittore:

mi pare d'essermi accorto che il tradurre così per esercizio vada veramente fatto innanzi al comporre, e o bisogni o giovi assai per divenire insigne scrittore, ma che per divenire insigne traduttore convenga prima aver composto ed essere bravo scrittore, e che in somma una traduzione perfetta sia opera più tosto da vecchio che da giovane (44).

Queste parole, con cui Leopardi pare quasi congedarsi dal suo giovanile apprendistato, non fanno che confermare quelle riserve che lo stesso Giordani, a suo tempo, aveva formulato relativamente al volgarizzare, da lui considerato - come già da Cicerone e Quintiliano (45) - niente di più che una pur valida esercitazione (46). Tuttavia, anche quando Giacomo ha di fatto accettato, dopo l'iniziale rifiuto, la posizione del più esperto studioso, la sua opinione sul tradurre non scadrà mai a mero livello didattico. Solo dopo la delineazione di un preciso progetto poetico, dunque ben oltre la stesura dei primi versi davvero originali, sui volgarizzamenti peserà l'a-

tuto dell'Enciclopedia italiana, 1987, p. 182: «erudizione, lingua e filologia venivano strumentalizzate (in modo spontaneo e necessario, e mediante una nuova e commossa apertura ai problemi dell'esistenza e della felicità) alla rivelazione di quella "integrale verità" lirica scrostata di ogni sovrastruttura culturale e di ogni "affettazione letteraria"». Tuttavia, in seguito, anche la traduzione, benché porti a una riappropriazione della incorrotta classicità, diviene sinonimo di affettazione: «il traduttore necessariamente affetta» $(Z i b .319,11$ novembre 1820) è però una constatazione, non una condanna che comporta una rinuncia (cfr. FASANO, Come gli antichi Greci cit., pp. 86-87).

(42) Zib. 1947 (19 ottobre 1821) e cfr. 2134-6 (21 novembre 1821).

(43) Zib. 1741 (19 settembre 1821).

(44) Lettera a P. Giordani del 29 dicembre 1817 (in LEOPARDI, Lettere cit., pp. 119-20)

(45) Cfr. Cic. De orat. 1, 155 e QuinT. Inst. 10, 5, 2.

(46) Giordani, nella lettera inviata a Giacomo il 12 marzo 1817, definisce la traduzione «esercizio che mi pare affatto necessario a divenir grande scrittore, e proprio all'età giovane» (in LEOPARDI, Epistolario cit., pp. 66-67). Cfr. FASANO, Come gli antichi Greci cit., p. 70: per il giovane si tratta di una concezione della «traduzione [...] come possesso e immedesimazione [...], accensione istantanea della scintilla poetica; per Giordani tradurre non è che il primo gradino di una lunga scalata verso la poesia». 
mara constatazione: «le antiche opere classiche, non solo perdono moltissimo, tradotte che sieno, ma non vaglion nulla, non paiono avere sostanza alcuna, [...] restano come stoppa e cenere» (47).

L'analisi della traduzione leopardiana del Moretum consente di mettere in luce da un lato i criteri generali che hanno guidato le scelte lessicali e linguistiche, dall'altro quelli che presiedono all'impalcatura stessa dei versi e alla successione delle parole. Per esplicita dichiarazione di Leopardi, la sfida è quella di creare un nuovo componimento che, pur ispirandosi all'antico, appaia dotato di vita propria e impreziosito da riferimenti intertestuali: in questo senso va intesa la libera traduzione che il volgarizzatore si prefigge (48). Siamo relativamente sicuri dell'orizzonte letterario in cui Leopardi collocava il Moretum: la citata attribuzione ad A. Settimio Sereno (o Severo), poeta dell'età flavia, lascia intuire un giudizio globalmente positivo, tanto più valorizzato dalla paternità greca dell'originale, da cui l'autore latino avrebbe tratto ispirazione. Più problematico definire quanto il traduttore abbia colto dei riecheggiamenti classici che oggi i critici riconoscono tra le pieghe del carme; attento lettore dei fiori più belli della letteratura antica, a cui ascriveva senza ombra di dubbio anche il «vago poemetto» (49), Leopardi potrebbe averne percepito i numerosi legami con la tradizione precedente: non soltanto con quella esplicitamente bucolico-georgica, di cui è emblema il virgiliano vecchio di Corico (50), ma anche con quella vena pastorale presente nei monumentali poemi ovidiani delle $\mathrm{Me}$ tamorfosi e dei Fasti (51).

$\mathrm{Al}$ di là dei precedenti classici, la traduzione leopardiana risente pure dei tentativi fatti da alcuni italiani del passato più o meno

(47) Zib. 3475 (19 settembre 1823).

(48) «Confesso senza rossore che ho tradotto questo poemetto alquanto liberamente, sì perché non ho giudicato necessario tradurlo colla fedeltà con che ho tradotto il primo Canto dell'Odissea, come perché non posso in buona coscienza adoperare una sola rima che non sembri spontanea» (dalla Nota introduttiva, poi non pubblicata, alla bella copia di a, in STEFANI, "La Torta" di Giacomo Leopardi cit., p. 146).

(49) Leopardi, Poesie e Prose cit., I, p. 548.

(50) Si veda a questo proposito almeno A. La Penna, Senex Corycius in Atti del Convegno virgiliano sul Bimillenario delle Georgiche - Napoli 17-19 dicembre 1975, Napoli, Istituto Universitario Orientale, 1977, pp. 37-66. Nello stesso filone rientrano gli amatissimi "Eo $\gamma \alpha$ esiodei, forse mai letti nell'originale greco, ma citati nel (pur presto ripudiato) Preambolo alla Titanomachia (1817) come luminoso esempio di naturale freschezza: cfr. LEOPARDI, [Preambolo alla] Titanomachia di Esiodo cit., $\$ \$ 10$ 14, pp. 36-38.

(51) Alludo all'episodio di Filemone e Bauci (Met. 8, 618-724) e a quelli di Celeo (Fast. 4, 507-562) e Irieo (Fast. 5, 493-535). 
recente; a questo proposito, è di nuovo Leopardi stesso a illuminarci quando cita Giuseppe Scaligero, il quale, oltre a pubblicare il Moretum e a ideare numerose congetture tuttora validissime, volse il testo latino in greco, pensando di restituirgli la sua veste originale; e, dopo lo Scaligero, una nutrita schiera di autori, dal Cinquecento in poi, si appassionarono al poemetto (52). Infine, al poeta di Recanati non era ignota un'ulteriore impresa, di più libera rielaborazione, risalente al chierico (dionisottianamente) Bernardino Baldi, vissuto a Urbino fra il 1553 e il 1617, e in seguito inserita nel volume della Crestomazia poetica italiana (1828) (53): la sua egloga Celéo, o l'orto richiama uno dei possibili modelli del Moretum, il vecchio Celeo, visitato da Demetra, di Ov. Fast. 4, 507-562, per poi subito affrancarsene (54). Il lavoro nei campi, la preparazione del pasto a base di polenta accompagnata dai prodotti dell'orto, per cui si invoca la protezione divina, confermano l'impressione, già leopardiana, dell'ingente debito contratto dal Baldi con l'autore del Moretum (55).

Tornando al volgarizzamento e prescindendo per il momento da modelli e precedenti, si individuano due orientamenti generali, fra loro opposti, nella prassi versoria del traduttore: Giacomo infatti tende ora a una semplificazione ora a una amplificazione dell'originale; mentre certe immagini sono ridotte, quando non del tutto annullate, altre risultano arricchite di dettagli, che, se non compaiono nel latino, nondimeno vi si adattano con straordinaria coerenza. Ecco un breve saggio della prima attitudine: excubitorque [...] ales (v. 2) > "e 'l gallo" (v. 2), con eliminazione della patina epicheggiante-militaresca, che però era presente in alcune varianti ("il vigil gallo" della prima stesura e "l'augel da la vegghia" del 1817) (56); et cinis obductae celabat lumina prunae (v. 9) > "[un tizzon] giacea sotto la cenere nascosto" (v. 15), dove scompaiono i bagliori della brace; gli altisonanti geminos [...] lacertos (v. 21) > "le braccia" (v.

(52) Cfr. Leopardi, Poesie e Prose cit., I, p. 548.

(53) Cfr. G. Leopardi, Crestomazia italiana. La poesia, a cura di G. Savoca, Torino, Einaudi, 1968, pp. 99-101. Del Celéo sono stampati da Leopardi solo i vv. 41128.

(54) Cfr. C. Laudani in Moretum cit., pp. 29-34. Leopardi amava molto le egloghe del Baldi e lo dichiarò apertamente in una nota d'autore alla canzone Sopra il monumento di Dante nell'edizione bolognese del 1824: esse «sono scritte con semplicità, candore e naturalezza tale, che in questa parte non le arrivano quelle del Sannazaro» (LeOPARDI, Poesie e Prose cit., I, p. 173).

(55) Cfr. Leopard, Poesie e Prose cit., I, pp. 548-549: «Chi ha letto il Celeo a buon diritto lodatissimo del Baldi, vedrà leggendo la Torta che questi due poemetti hanno la stessa andatura e paiono fatti ad una stampa».

(56) Cfr. Stefani, "La Torta" di Giacomo Leopardi cit., p. 149. L'incipit è certamente tra i punti più tormentati dell'intero volgarizzamento quanto a lavorio compositivo. 
31); la pulizia della macina è ridotta all'osso, perverrit cauda silices gremiumque molarum (v. 23) > "il mulinello appresta" (v. 32); numerosi sono i casi di espressioni per nulla tradotte, traiectus medium sparto (v. 57), varias disponere plantas (v. 68) o, nella rassegna degli ortaggi, inulae (v. 72), siser (v. 73), intiba (v. 84) (57); dei tre elenchi, soltanto quello contenente gli ingredienti del moretum è riprodotto fedelmente; a questo proposito non va dimenticata «l'omissione di aggettivi specifici» (58), riferiti in particolare alle specie vegetali con tono realistico: cepa rubens (v. 82) > "cipolla" (v. 113), comas apii graciles (v. 88) > "la cima / [...] de l'appio" (vv. 118-119), rutamque rigentem (v. 88) > "ruta" (v. 118), coriandra trementia (v. 89) > "coriandoli", ma fecundusque rumex (v. 72) > "e '1 romice fecondo" (v. 99), sectique [...] porri (v. 82) > "vil porro" (v. 113) (59). Il finale, nel latino, è arricchito di una ulteriore scena di vestizione epicheggiante tutta giocata sull'ambiguità di ocrea e galerus, l'uno evocante i robusti schinieri degli eroi omerici e virgiliani (e qui impiegato per miseri gambali), l'altro usato dagli antichi Latini anche come elmo (qui a indicare un semplice berretto a protezione della testa dai rigori del freddo) (60): Leopardi non asseconda una simile caratterizzazione e fa indossare al contadino "stivali" e "cappel" (v. 160), mentre il particolare degli iuga [...] lorata (v. 121), un hapax (61), è da lui trascurato; «appare evidente quanto la genericità letteraria del linguaggio settecentesco del giovane traduttore contribuisca a dissolvere la suggestione di un abbigliamento specifico di una tecnica o mestiere contenuta nell'intero verso latino» (62).

Di segno opposto quelle che possiamo definire amplificazioni, ovvero integrazioni che il traduttore giudica opportuno inserire quasi proseguendo il sentiero avviato dall'anonimo: alle azioni di esplorazione delle tenebre e di ricerca del fuoco, svolte da Simulo al suo risveglio, Leopardi aggiunge "va passo passo" (v. 8), cioè cammi-

(57) L'omissione di radix (v. 75) è invece dovuta alla assenza del verso, lacunoso, nelle edizioni che il traduttore aveva a disposizione a Recanati.

(58) L. Alboreto, Note alla traduzione leopardiana del Moretum, in «Atti dell'Istituto Veneto di Scienze, Lettere ed Arti», CXXXVI, 1977-8, p. 483.

(59) Si tratta di una specie particolare di porro a cui si tagliavano le foglie durante la crescita (al v. 73 compaiono invece i porra capitata, che venivano fatti crescere con le foglie; cfr. A. Perutelli in [P. Vergili Maronis] Moretum cit., p. 119): qui C. Morelli, Note sul Moretum, in «Rendiconti della Reale Accedemia dei Lincei. Classe di Scienze morali, storiche e filologiche», XXIII, 1914, p. 80 vede un indizio utile alla cronologia del componimento: questa tipologia di porri, infatti, molto rinomata in età neroniana (come attesta PLIN. 19, 108-110), ai tempi di Giovenale era invece considerata degna dei poveracci (Iuv. 3, 293)

(60) Cfr. rispettivamente Verg. Aen. 7, 634 (levis ocreas lento ducunt argento) e 7, 688-9 (fulvosque lupi de pelle galeros / tegmen babent capiti).

(61) Cfr. A. Perutelli in [P. Vergili Maronis] Moretum cit., p. 144.

(62) Alboreto, Note alla traduzione leopardiana del Moretum cit., p. 481. 
nando adagio (Battaglia), secondo la lezione dantesca (63); la menzione della "sera" del giorno prima (v. 13) cozza con la tendenza, nell'anonimo, a non rimandare mai a un tempo ulteriore rispetto a quello della narrazione; lumen (v. 14) > "l lume infermo" (v. 22); "men timido e più franco indi s'avanza" (v. 23) è una vera e propria aggiunta che si può collocare nel testo latino tra il v. 14 e il v. 15; fusca colore (v. 33) > "sua faccia di color pressoché nera" (v. 48), con la giusta intuizione dell'utilizzo di color propriamente per «l'incarnato del viso» (64); iamque subactum / opus palmisque suum dilatat in orbem / et notat impressis aequo discrimine quadris (vv. 4648) > "poi ch'assodata fu la facil massa, / ei co le palme a dilatarla imprende, / appianala, rotondala, l'abbassa, / la segna in quadri uguali e la distende / e la compone" (vv. 67-71); ac remanent summo purgamina dorso, / subsidit sincera foraminibusque liquatur / emundata Ceres (vv. 40-42) > "e sopra / restan le grosse Cereali spoglie, / mentre purgato in sottil pioggia il grano / cade pe' fori in sul sopposto piano" (vv. 57-60), dove Leopardi aggiunge l'immagine del piano, su cui si rovescia il frumento mondato, un dettaglio che il latino inserisce dopo (levi [...] tabula, vv. 42-43, tradotto comunque in "liscia tavola", v. 62) (65); verum bic non domini (quis enim contractior illo?) / sed populi proventus erat (vv. 77-79) > "ma questi cibi il povero padrone / raro a la bocca d'appressare ardia" (vv. 103$104)$, in cui Leopardi tralascia la parentetica, ma aggiunge il dettaglio della bocca; nel finale, in segetes (v. 122) > "pe' campi suoi" (v. 162), «dei quali nel testo latino non v'è traccia alcuna [e che] circondano l'exigui cultor rusticus agri [...] di una gloria di latifondismo del tutto fuori luogo» (66). Rientra in parte in questa seconda attitudine la capacità che il traduttore ha di rendere ancora più vive, se possibile, le immagini dell'originale: il realismo di certi dettagli, già ampiamente messi in rilievo dall'anonimo, è così perfettamente assecondato. Un esempio patente viene dall'accensione della lucerna: "volgesi il buon villano e dà di piglio / a la lucerna e 'n giù la piega, e chino / co l'ago slunga l'arido stoppino” (vv. 16-17) (67). Azzeccatissimo l'aggettivo "moribondo" (v. 19) per il languentem ignem (v. 12), "ch'al fin chiarisce" (v. 20); al verbo "chiarire", molto amato nel Cinquecento e impiegato in senso proprio soprattutto

(63) Inferno XXIX 70: "passo passo andavam sanza sermone".

(64) C. LAUDANI in Moretum cit., p. 71.

(65) Cfr. I. MazzinI, Le traduzioni poetiche giovanili di Giacomo Leopardi (180917). Differenziazioni rispetto al modello, in Leopardi e la cultura europea. Atti del Convegno internazionale dell'Università di Lovanio - Lovanio 10-12 dicembre 1987 (a cura di F. Musarra, S. Vanvolsem, R. Guglielmone Lamberti), Leuven-Roma, University Press-Bulzoni, 1989, p. 338.

(66) C. Vossler, Leopardi, trad. it. di T. Gnoli, Napoli, Ricciardi, 1925, p. 82.

(67) "Slunga la spada" è in Marino, Adone XIV 392. 
per il dì, Leopardi pare qui assegnare valenza intransitiva nel senso di "illuminarsi, divenir chiaro" (68).

Nella stessa direzione va la caratterizzazione dell'orto quale bobby di Simulo nei giorni piovosi o festivi: "quest'era il suo conforto / ne' tempestosi dì, ne' dì di Festa, / quand'arar non potendo, era costretto / di starsi neghittoso entro "1 suo tetto" (vv. 87-90); saepe viri nares acer iaculator apertas / spiritus (vv. 105-106) > "spesso l'acuto odor saetta il naso / che si raggrinza, al povero villano" (vv. 139-140), con l'aggiunta dell'incresparsi del viso facilmente immaginabile per via dell'acre olezzo dell'aglio. In quest'ultimo caso si tratta però di una suggestione pariniana: "e le narici / schifo raggrinza" (Il Mezzogiorno, vv. 487-8, un passo tutto pervaso dai riecheggiamenti parodistici dell'epica e dei suoi eroi, impavidi contro i morsi della fame proprio come Simulo).

Tipico di Leopardi è poi il trasferimento di attribuzioni da un termine all'altro rispetto al latino: hortus erat iunctus casulae (v. 60) $>$ "giacea presso la casa un piccol orto" (v. 85), dove, forse riprendendo il successivo exiguus spatio (v. 62), le ristrette dimensioni sono qualità non più della casa, bensì dell'orto; ergo aliam molitur opem sibi providus heros (v. 59) > "il villan saggio / ad altra cosa volge il suo coraggio" (vv. 83-84), con il recupero della apparentemente caduta coloritura militaresca di Simulo (già lodato per il suo "provvido consiglio", v. 14) attraverso la menzione del "coraggio".

Inoltre, si può notare una certa libertà nell'ordine di termini e concetti anche laddove uno stravolgimento non appariva necessario; ad esempio, mentre nel latino il ricco che chiede le eccedenze al povero è menzionato prima dei giorni piovosi e festivi, nel volgarizzamento questa successione risulta invertita: interdum locuples a paupere plura petebat. I Nec † sumptus erat ullius opus sed regula curae: / si quando vacuum casula pluviaeve tenebant / festave lux (vv. 6467) > "quest'era il suo conforto / ne' tempestosi dì, ne' dì di Festa, [...] e spesso / cose assai di quell'orto richiedea / al povero cultore il ricco istesso" (vv. 87-88/92-94). Un simile artificio culmina nell'elenco dei prodotti dell'orto: in quello dei vegetali destinati alla vendita, "la zucca" (v. 97), che in latino chiude la rassegna, nel volgarizzamento la avvia, mentre "'l cavolo" (v. 100), che in latino compare per primo, è da Leopardi collocato quasi in fondo. Da notare ancora, nella versione italiana, la tipica costruzione "a epifrasi", carissima al poeta, in "lunghe rughe e spesse" (v. 52) e "duro cacio e bianco" (v. 127), dove la normale collocazione del secondo aggettivo, in un sintagma nominale non-marcato, è sostituita dal suo iso-

(68) Cfr. M.M. BoIARdo, Orlando innamorato I 15 55: «né ancora era chiarito in tutto il giorno». Ma si tratta di un uso già dantesco (cfr. Paradiso IX 15) e ripreso da Leopardi ne Il sabato del villaggio, v. 37. 
lamento oltre la congiunzione coordinante, in posizione di forte rilievo espressivo (69).

Un discorso a parte meritano i numerosi latinismi che, come in tutte le versioni poetiche leopardiane, colorano qua e là la traduzione ora alleggerendola ora appesantendola: "rustico cultore" (v. 3), che rende letteralmente l'espressione latina, "parton [l'opra]" (v. 33, con una coincidenza tra l'uso transitivo arcaico-letterario e popolare-toscano del verbo), "mamme" (v. 50), "tepid'onda" (v. 63), "mondò" (v. 73), "contesta" (v. 86) (70), "grave" (v. 108) nel senso di "pesante", "ito" (v. 143). "[Chiome] tortuose" (v. 47) è pure oggetto di una chiosa specifica nello Zibaldone: «nomi in uosus ec. Impetuosus, da impetus us. Se quella voce, e impetuose, non sono veramente nel buon latino (v. Forcell.) certo elle sono nelle lingue figlie. (V. il Gloss.) Tortuosus, tortuose ec. da tortus us» (71). In tale ottica va forse inquadrato anche il titolo trascelto da Leopardi per il suo volgarizzamento; il traduttore deve essersi interrogato sul possibile nesso tra il nome del moretum e la sua forma: "Torta" (che compare anche al v. 153) sembra non solo un sostantivo appropriato all'aspetto finale del composto, ma un nome che rivela, attraverso la sua etimologia, un legame con la preparazione: il latino tardo torta indica infatti la "pasta ravvolta" (Devoto-Oli), benché il moretum, di per sé, sia in realtà una sorta di pesto, il quale, soltanto dopo la sua unione con il pane, può dar luogo alla forma suggerita dal sostantivo leopardiano (72).

(69) Basti pensare al celeberrimo "le magnifiche sorti e progressive" (La ginestra o il fiore del deserto, v. 51, con voluta inversione delle parole del Mamiani e messa in rilievo dell'aggettivo forse più irriso dall'ultimo Leopardi, per cui cfr. S. TIMPANARO, Note leopardiane in Idem, Aspetti e figure della cultura ottocentesca, Pisa, Nistri-Lischi, 1980 , pp. 287-93). In Leopardi, in particolare, tale successione, tipica della lingua antica e letteraria, sembra il punto di partenza per sviluppi più articolati come in "gli studi leggiadri / talor lasciando e le sudate carte" (A Silvia, vv. 15-16): qui si ha epifrasi vera e propria, ovvero iperbato, ma tra due membri in rapporto di coordinazione. Entrambi i costrutti erano già nel Baldi; così nel suo Celéo: "al famelico ventre ed importuno" (v. 40) e "che dal sudore ei s'ebbe e da la polve" (v. 54). Su questo aspetto cfr. almeno la Grammatica dell'italiano antico, a cura di G. Salvi - L. Renzi, I, Bologna, Il Mulino, 2010, pp. 268 e 601.

(70) Nella tradizione letteraria "contesto, -a" è impiegato per pietre preziose incastonate in armi oppure per legni o tessuti particolari; la stessa immagine (del vimine e del suo impiego) compare invece in Ariosto che, a proposito dell'allestimento di fortificazioni, scrive: "Già scale innumerabili per questo / da' luoghi intorno avea fatto raccorre, / et asse e travi, e vimine contesto, / che lo poteano a diversi usi porre" (Orlando furioso XIV 67); ma il costrutto viminibus contexta risale almeno a CAes. Gall. 6, 16, 4.

(71) Zib. 3617 (6 ottobre 1823).

(72) Quanto alla reale etimologia del latino moretum, di fronte a una serie di insoddisfacenti proposte, l'ipotesi più verosimile sembra quella di una mera traslitte-

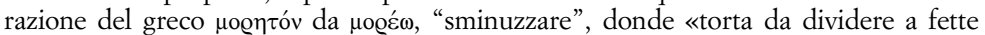
o a spicchi; o anche, se vuolsi, torta fatta con erbe sminuzzate e pestate» (C. PASCAL, Il poemetto Moretum, in «Athenaeum», I, 1913, p. 166). Inoltre, cfr. A. PERU- 
In definitiva, se l'analisi delle varianti mostra che, dalla prima all'ultima stesura, Leopardi optò per il latinismo soltanto quando l'italiano non gli forniva spunti adeguati, nondimeno la scelta di un vocabolario fortemente legato all'originale non va troppo meccanicamente intesa in direzione antiaulica (73). È piuttosto - come si vedrà tra poco - il legame con la tradizione letteraria a guidare il giovane traduttore verso prestiti dotti: i nomi del Poliziano, del Baldi, del Tasso, dell'Alamanni e di tanta produzione pastorale dal Cinquecento al Settecento gravavano sulle sue gracili spalle di giovane della provincia marchigiana, la cui memoria poetica era imbevuta di classici, e non solamente di classici desanctisianamente concepiti. Nella medesima prospettiva si pongono i vari toscanismi, ora etimologici ("Affricana", v. 44, "cammino", v. 79, "maladice”, v. 144) (74) ora relativamente all'uso ("parton [l'opra]" v. 33, con accezione transitiva): quando non corrispondono all'uso popolare, essi, al pari, ma forse più dei latinismi, rafforzano il carattere letterario del carme.

In generale, sembra che l'atteggiamento a tratti irridente verso il filone bucolico e georgico, ma anche verso l'epica assunto dall'anonimo (75) non sia stato recepito da Leopardi, che pure era stato attento lettore tanto di Ovidio quanto delle Georgiche virgiliane (76).

Telli in [P. Vergili Maronis] Moretum cit., pp. 141-2 e soprattutto A. Walde-J.B. Hofmann, Lateinisches Etymologisches Wörterbuch, II, Heidelberg, C. Winter, 1954³ p. 112, che contiene un piccolo campionario delle principali proposte in merito; l'altra possibilità, molto seguita dalla critica, consisterebbe nel presupporre una connessione etimologica tra moretum e mortarium - a questo proposito, si veda, tra gli altri, D.O. Ross, JR., The Culex and Moretum as Post-Augustan Literary Parodies, in «Harvard Studies in Classical Philology», LXXIX, 1975, p. 260. L'etimologia da $\mu$ o-

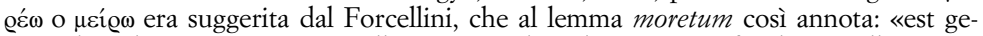
nus cibi, idem ac intritum et alliatum, quod ex his octo conficiebatur, allio, apio,

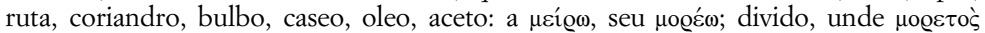
divisus: etenim ex praedictis rebus edulibus sectis, et pistillo operose tritis, minutis, coactisque in mortario moretum conficitur» (cito da Totius Latinitatis Lexicon opera et studio Aegidii Forcellini Seminarii Patavini Alumni lucubratum et in hac editione post tertiam auctam et emendatam a Iosepho Furlanetto eiusdem seminarii alumno novo ordine digestum amplissime auctum atque emendatum cura et studio doct. Vincentii De-Vit eiusdem seminarii alumni ac professoris, IV, Prati, Typis Aldinianis, 1868, p. 176).

(73) Di questo avviso Stefani, "La Torta" di Giacomo Leopardi cit., p. 173.

(74) Su maladice cfr. G. Boccaccio, Comedia delle ninfe fiorentine, VI ed Elegia di madonna Fiammetta, 3, 5, ma anche Appressamento della morte, Canto Terzo, v. 154: "e Guerra maladice e la sua possa".

(75) Su cui cfr. specialmente Ross, JR., The Culex and Moretum cit., pp. 235-63. Così si pronuncia in proposito AlBorETo, Note alla traduzione leopardiana del Moretum cit., p. 487: «ironia ed epicità si possono definire i due poli tra cui oscilla il riferimento di una realtà umile a una tradizione solenne nel Moretum [...]. Ora, la Torta leopardiana, in linea di massima, lascia cadere siffatta oscillazione».

(76) Cfr. S. SconocchiA, La cultura classica nelle Marche del primo Ottocento e la filologia di Giacomo Leopardi, in Quei monti azzurri. Le Marche di Leopardi (a cura 
D'altra parte, quella tendenza a chiamare con il giusto nome le cose, segno di un contatto diretto con la natura da parte di Simulo, permane nel volgarizzamento, dove, se le attribuzioni sono lievemente sfrondate, i componenti della macina, le specie vegetali e così via trovano adeguata collocazione.

Da ultimo, un fattore "tecnico" esercitava un forte condizionamento: la struttura metrica scelta per la traduzione, con un'alternanza di rime rigorosissima, non lasciava ampi margini di libertà creativa sul piano della selezione delle parole; lo stesso Leopardi ne era consapevole se candidamente confessava: «non posso in buona coscienza adoperare una sola rima che non sembri spontanea» (77), in cui, se non altro, sono lasciati intuire, senza giudizi di valore, $\mathrm{i}$ vincoli formali a cui soggiacere.

Quanto ai possibili riecheggiamenti contenuti nella traduzione, un ruolo di rilievo è occupato dai volgarizzatori precedenti (78). Non mancano relazioni con l'egloga di Bernardino Baldi, che Giacomo certamente aveva riletto in vista della sua traduzione: "cultor" (v. 6) > "cultore" (v. 3); il dotto latinismo "[di prun] contesta [siepe]" (v. 25) > "[di vimini] contesta [fratta]" (v. 86); "[uom che] neghittoso [il dì non mena]" (v. 37) > "[di starsi] neghittoso [entro '1 suo tetto]" (v. 90); "arido [fomento]" (v. 47) > "arido [stoppino]" (v. 18); "purgata [farina]" (v. 76) > "purgato [il grano]" (v. 59).

L'atmosfera bucolica in cui i pastori con i loro figli custodiscono il gregge e cantano felici la tranquillità della loro vita è delineata da Tasso nella Gerusalemme liberata (VII 10):

\footnotetext{
Altrui vile e negletta, a me sì cara

che non bramo tesor né regal verga,

né cura o voglia ambiziosa o avara

mai nel tranquillo del mio petto alberga.

Spengo la sete mia ne l'acqua chiara,

che non tem'io che di venen s'asperga,

e questa greggia e l'orticel dispensa

cibi non compri a la mia parca mensa.
}

Se certo l'autore del Moretum non aveva alcuna pretesa né volontà di dar vita ad un quadretto bucolico, così Leopardi deve aver percepito il poemetto, affiancato alle illustri lodi della vita agreste; l'autosufficienza alimentare del pastore incontrato dalla tassiana Er-

di E. Carini-P. Magnarelli-S. Sconocchia), Venezia, Marsilio, 2002, p. 286 e P. TuSCANO, Leopardi lettore delle "Georgiche", in Leopardi e il mondo antico. Atti del V Convegno internazionale di studi leopardiani - Recanati 22-25 settembre 1980, Firenze, Olschki, 1982, pp. 621-31.

(77) Dalla Nota introduttiva, poi non pubblicata, alla bella copia di a, in STEFANI, "La Torta" di Giacomo Leopardi cit., p. 146.

(78) Di questo problema si è già occupato, seppur non in modo organico, ALBORETO, Note alla traduzione leopardiana del Moretum cit., pp. 471-89, in particolare nelle note. 
minia, la sua esclusione dal mondo, la gioia del poco compaiono anche nella caratterizzazione di Simulo e di alcuni personaggi evocati nei Canti: la "parca mensa" è parimenti quella dello "zappatore" de Il sabato del villaggio, v. 28 (79).

Fuori di un contesto prettamente bucolico, la caratterizzazione di Cibale, la schiava africana di Simulo, pare delineata sulla scia di un passo della traduzione dell'Eneide di Annibal Caro, dedicato alla descrizione di Achemenide, compagno d'Ulisse e ritrovato da Enea in Sicilia in uno stato di indicibile trascuratezza:

Già del giorno seguente era il mattino,

e ' 1 chiaro albore avea l'umido velo

tolto dal mondo, quando ecco dal bosco

ne si fa 'ncontro un non mai visto altrove

di strana e miserabile sembianza,

scarno, smunto e distrutto: una figura

più di mummia che d'uomo (80).

Del resto, una «vecchia fante» ministra cibo e bevanda anche al vecchio Laerte, che abita «in villa», nel Saggio di traduzione dell'Odissea, Canto primo, vv. 257-263.

Inoltre, almeno una delle operazioni svolte da Simulo, quella del setacciare ("l'abburatta", v. 57), è presente nel fr. 7 Pellizer-Tedeschi di Semonide, tradotto da Leopardi nel 1823, ed elencata tra le

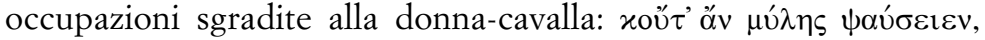

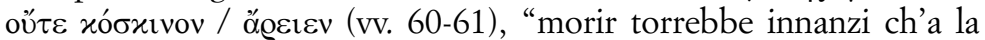
macina / por mano, abburattar, trovare i bruscoli” (vv. 56-57). La purificazione del grano richiama invece una similitudine iliadica ben tradotta dal Monti, a cui il Nostro si potrebbe essere ispirato:

Come allor che di Zefiro lo spiro

disperde per le sacre aie la pula,

mentre la bionda Cerere la scevra

dal suo frutto gentil, che il buon villano

vien ventilando; lo leggier spulezzo

tutta imbianca la parte ove del vento

lo sospinge il soffiar: [...] (81).

Fu sempre all'interno del prevalente filone tematico bucolico-pastorale che Leopardi, nel 1827, avviò l'allestimento di una antologia della poesia italiana (accanto a quella della prosa edita nello stesso anno). Affrontando l'ardua impresa di cernita, il poeta attinse a piene mani al patrimonio, anche meno noto, della tradizione letteraria italiana e mostrò una sensibilità particolare per quei testi dedicati

(79) Cfr. M. M. Lombardi in G. Leopardi, Canti, a cura di F. Gavazzeni e M. M. Lombardi, Milano, BUR, 1998, p. 470.

(80) A. Caro, Eneide III 926-32.

(81) V. MonTI, Iliade V 657-63. 
alla vita agreste o aventi uno sfondo rustico analogo a quello del Moretum. Leopardi, una volta individuato il brano, lo forniva di un titolo secondo il proprio arbitrio e decideva se pubblicarlo integralmente $\mathrm{o}$, più spesso, in parte (82): solo per fare qualche esempio, vi troviamo, oltre alla citata egloga del Baldi, Spettacoli della campagna (dalle Stanze del Poliziano), Canto funebre pastorale (dall'Arcadia del Sannazaro), Esortazione all'agricoltore perché s'industrii di migliorare lo stato del suo terreno (dalla Coltivazione dell'Alamanni), Amori pastorali (di Benedetto Varchi), Lodi della vita rustica (dal Podere di Luigi Tansillo), e così via (83). La memoria poetica di Leopardi, già all'altezza cronologica della nostra traduzione, e ancor più alla fine degli anni Venti, doveva essere a tal punto imbevuta di testi in prosa e poesia dalle provenienze più disparate $\mathrm{da}$ rendere oggi ardua l'individuazione di un singolo e preciso modello per i suoi versi.

Resta costante e vivo l'interesse per la vita di campagna, non solo quale teatro di amori bucolici e certami canori, sulla scia degli idilli di Mosco, ma anche sotto il profilo più concreto e materiale, legato alla coltivazione dei campi, all'aumento di produttività, all'amministrazione della villa, al rapporto con i vicini. Cultori e villani rustici, italici, lieti con i loro alberghi e ostelli disadorni, gli orticelli che li rendono indipendenti dal mondo esterno dominano molti dei passi "pastorali" inseriti da Giacomo nella sua Crestomazia e si pongono tutti sulla scia di Simulo, che troneggia come loro progenitore latino, se non addirittura greco.

Alcuni motivi tematici contenuti nel poemetto latino sembrano colpire da vicino la sensibilità del traduttore al punto da ritornare, anche a distanza di tempo, in alcuni dei Canti. Non va certo esagerata la loro primogenitura in seno al Moretum, visto che certe situazioni sono ampiamente diffuse e attestate in altri passi della letteratura italiana, prima che latina o greca; tuttavia, è interessante notare come Leopardi abbia spesso scelto di volgere nella propria lingua madre componimenti ispirati, in un modo o nell'altro, alle situazioni che più di tutte egli considerava poetiche.

Innanzitutto occorre puntualizzare, se non proprio il genere let-

(82) Talvolta l'antologista interveniva con mano pesante sul brano trascelto «in base a un'idea di restauro che si avvicina pericolosamente al rifacimento» (G. BoLLATI, Giacomo Leopardi e la letteratura italiana, a cura di G. Panizza, Torino, Bollati Boringhieri, 1998, p. 83).

(83) «Le colorite sestine della Torta ricordano sovente l'ampia letteratura georgico-pastorale e venatoria dell'età laurenziana o immediatamente successiva» (ORLANDO, Il pessimismo antico cit., p. 920). 
terario, almeno l'atmosfera che, a parere di Leopardi, fa da cornice al Moretum; il fatto che questo testo fosse definito idilio attribuito a Virgilio nell'edizione padovana che conteneva anche il Celéo di Baldi conferma un'impressione che già da sé il poeta poteva aver avuto; a ciò si aggiunga la definizione che Macrobio aveva dato dell'omonimo componimento di Sueio, idyllium, appunto (84). Lo stesso Leopardi, in una nota linguistica sul verbo popolareggiante transverso, presente nel Moretum, avrebbe annotato:

ora ecco questa parola barbara in un gentilissimo poemetto o idillio del secolo di Augusto o del susseguente, dico in quel poemetto che s'intitola Moretum, (attribuito da alcuni a Virgilio, da altri ad un A. Settimio Sereno o Severo, poeta Falisco del tempo de' Vespasiani) (85).

Al di là di un diffuso realismo e di qualche dettaglio volgare, il carme delinea un bozzetto di vita quotidiana, ben diverso certo dagli idilli di Teocrito, Mosco e Bione, apprezzati e tradotti da Leopardi nel 1815, ma non per questo meno suggestivo. Si riscontra da un lato l'assenza dell'amore, nelle sue pur minime sfaccettature - a parte il riferimento ai poteri afrodisiaci della ruchetta - dall'altro, una generale struttura da idillio pastorale, a cominciare dall'incipit, che ricorda un ben più dolce risveglio in Europa, vv. 1-7, dove il già dell'apertura svolge quella stessa funzione del corrispondente iam del v. 1 del Moretum, lasciato però cadere nella Torta insieme con il carattere parodistico che esso doveva rivestire se accostato a certe descrizioni omeriche (86):

Già Venere ad Europa, della notte

nella terza vigilia, allor che omai

era presso il mattino, un dolce sogno

mandò, quando il sopor sulle palpebre

più soave del miel siede, e le membra

lieve rilassa, ritenendo intanto

in molle laccio avviluppati i lumi.

(84) Cfr. Macr. Sat. 3, 18, 11 e cfr. Leopardi, Poesie e Prose cit., I, p. 548: «questi [Svevio] pur fece un Moretum che però certi versi allegatine da Macrobio fan chiaro essere stato altro dal nostro». In generale, per un'analisi a tutto tondo degli idilli leopardiani e dei loro motivi conduttori anche alla luce delle varianti si veda M. SANTAgata, Quella celeste naturalezza. Le canzoni e gli idilli di Leopardi, Bologna, Il Mulino, 1994, pp. 135-69.

(85) Zib. 1143. Sullo stesso verbo transversare Leopardi si era già soffermato in Zib. 107, dove aveva citato il poemetto pseudo-virgiliano. Si vedano G. LEOPARDI, Scritti filologici (1817-1832), a cura di G. Pacella e S. Timpanaro, Firenze, Felice Le Monnier ("Scritti di Giacomo Leopardi inediti o rari", VIII), 1969, pp. 121-2 e TIMPANARO, La filologia cit., pp. 57 e 225.

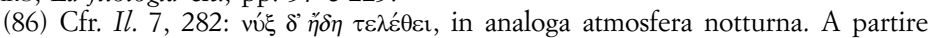
dall'età augustea poeti e scrittori (come SEN. Epist. 122, 11-13) si fecero beffa di tali formule per la descrizione di albe e tramonti, tanto altisonanti quanto inconsistenti. 
Il risveglio di Simulo è descritto in modo meno mellifluo, ma non più scarno: scompare il sogno, che rientra nella trama narrativa di Europa (e forse sarebbe risultato troppo poetico e poco adatto ad un contadino affamato); tuttavia, l'avvio del brano ha come sfondo l'alba ed è scandito dall'allontanamento del torpore notturno ("sopor", v. 4 > "sopor", v. 6) e dal risveglio delle membra addormentate.

Andamento molto simile aveva un'altra egloga del Baldi, la quindicesima, Mopso o l'epitafio; nella parte iniziale vi si legge:

già l'altezza maggior del sommo cielo
varcata avéa la notte, e'n ver' l'occaso
traéa tacita seco i sogni e l'ombre,
torbida schiera, paventosa e lieve;
quando, lasciate l'oziose piume,
il vecchio Mopso, dal dolor compunto
de l'acerba memoria di quel giorno
che 'l suo caro signor tolse di vita,
uscì fuor de l'albergo, [...] (87).

Rientrava nel genere idillico pure la menzione di orti e piante in essi coltivate, come nel Canto funebre di Bione bifolco amoroso, vv. 140-141, citati con qualche variante nel Discorso intorno alla poesia romantica come esempio di «celeste naturalezza» (88): "poi che morìr negli orti, / le malve, o l'appio verde, o il crespo aneto" ("crespo aneto", v. 81, è appeso sul focolare di Simulo, mentre "malve", v. 98, e "appio", v. 119 sono nel suo orto). Non va poi dimenticata una pur grezza vocazione canora di Simulo, il quale rustica carmina cantat (v. 29, con figura etimologica e allitterazione), tradotto da Leopardi "rozzo canto rusticano" (v. 41), con fusione nella medesima immagine dei rustica carmina e della vox agrestis (89). In definitiva, sotto molteplici aspetti, il Moretum rivela un legame con gli idilli dell'antichità; questo non significa che sia giusto ascriverlo a quel genere letterario né che il suo anonimo autore avesse una simile pretesa, ma che in questa veste lo leggeva, nella quiete del suo palazzo, Leopardi. A pochi anni dopo, a partire dal 1819, risalgono i suoi Idilli, «esprimenti situazioni, affezioni, avventure storiche del mio animo» (90) e inaugurati da L'infinito: essi si differenziano

(87) B. BALDI, Egloga decimaquinta. Mopso o l'epitafio, vv. 13-21. E cfr. la prima strofa del Celéo, citata per esteso da Leopardi nella nota (2) della Torta: «Sparir vedeasi già per l'Oriente / Qualche piccola stella e spuntar l'alba; / Già salutar il giorno omai vicino / s'udia col canto il coronato augello, / Quando pian pian dal letticciuolo umile / Celeo, vecchio cultor di pover orto, / alzò desto dal sonno il pigro fianco» (LeOpardi, Poesie e Prose cit., I, pp. 548-9).

(88) Cfr. Leopard, Poesie e Prose cit., II, pp. 403-4.

(89) Cfr. F. D’Intino in Leopardi, Poeti latini e greci cit., p. 305.

(90) G. LeOpardi, Disegni letterari XII. 
certamente dai loro omonimi latini e greci, tuttavia alcuni elementi permangono immutati. L'inizio del dì, ad esempio, scandisce l'apertura de La vita solitaria (e de Il sogno), vv. 1-8, dove "la capanna" sembra un calco della casula di Simulo (91):

La mattutina pioggia, allor che l'ale

battendo esulta nella chiusa stanza

la gallinella, ed al balcon s'affaccia

l'abitator de' campi, e il Sol che nasce

i suoi tremuli rai fra le cadenti

stille saetta, alla capanna mia

dolcemente picchiando, mi risveglia;

e sorgo $[\ldots]$.

Nello stesso idillio è poi presente un'immagine tanto cara a Leopardi, quella del canto durante il lavoro:

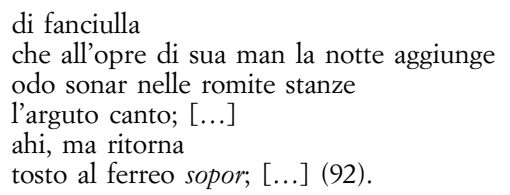

Si tratta di una suggestione che ritornerà vivissima in A Silvia, vv. 7-11: "sonavan le quiete / stanze, e le vie dintorno, / al tuo perpetuo canto, / allor che all'opre femminili intenta / sedevi”. Benché in entrambe le liriche la protagonista sia una donna e la melodia che ne scaturisce ben più soave, la funzione del canto resta la medesima anche nel caso di Simulo: alleviare le fatiche del lavoro quotidiano, al telaio nel caso delle donne (e dei loro modelli classici), (93) alla macina in quello di Simulo (vv. 41-42):

e con suo rozzo canto rusticano

alleggia sua fatica il buon villano.

Il traduttore impiega qui un verbo dantesco (cfr. Inferno XXII

(91) Una "povera capanna" era anche la casa di Micone ne Le rimembranze (v. 2), idillio del 1816, poi ripudiato dall'autore. Ma già nella canzonetta puerile La campagna (1809), che rivela un gusto molto precoce per la tematica rusticano-bucolica, fa la sua comparsa "l'umil tugurio" dei contadini, non toccato dal fulmine che invece colpisce il palazzo dei ricchi (vv. 29-32).

(92) G. LEOPARDI, La vita solitaria, vv. 63-68.

(93) Cfr. Verg. Georg. 1, 293-4, e Aen. 7, 11-14, «quel passo del canto di Circe come pregno di fanciullesco mirabile e da me amato già da scolare» (LEOPARDI, Poesie e Prose cit., II, p. 1190 e cfr. pp. 393-4 e Zib. 1930, 16 ottobre 1821). Tali versi virgiliani, dietro i quali si celano le omeriche Circe, Penelope, Calipso, Andromaca, sono stati indicati come modelli di A Silvia, vv. 7-11 da L. BLAsuCCI, Lo stormire del vento tra le piante: testi e percorsi leopardiani, Venezia, Marsilio, 2003, pp. 133-4 (cfr. inoltre BRUNI, Il canto della donna al telaio cit., pp. 7-13). 
22), già sfruttato nella canzone Sopra il monumento di Dante, v. 184: "quel mirto / ch'alleggiò per gran tempo il nostro male"; lo aveva fatto proprio anche Annibal Caro nella sua Eneide ("[la notte] '1 pensier [non] alleggia", IV 818) e se ne servì a più riprese Tasso quasi sempre coniugandolo al concetto di dolore (94).

Il rustico ritornello del contadino sarebbe degno piuttosto del "solitario canto / dell'artigian" de La sera del di di festa, "che riede a tarda notte, / dopo i sollazzi, al suo povero ostello" (vv. 25-27); e de La quiete dopo la tempesta: "l'artigiano a mirar l'umido cielo, / con l'opra in man, cantando, / fassi in su l'uscio" (vv. 11-13). Per un contesto rustico più affine a quello del poemetto occorre aprire Alla sua donna, vv. 34-35: "per le valli, ove suona / del faticoso agricoltore il canto", contrapposto, come in A Silvia, alla tristezza in cui si trova immerso l'io poetico (95). Ma già nei Ricordi d'infanzia e di adolescenza Leopardi aveva annotato di aver udito «il canto di quel muratore mentr'io componeva» (96) e, nella lettera in cui racconta al fratello Carlo la visita al sepolcro romano del Tasso, Leopardi scrive che la strada diretta alla chiesa di S. Onofrio «è tutta costeggiata di case destinate alle manifatture, e risuona dello strepito de' telai e d'altri tali istrumenti, e del canto delle donne e degli operai occupati al lavoro» (97). Le percezioni auditive hanno nel Nostro la potenza di scatenare un'evocazione spesso dolorosa:

Dolor mio nel sentire a tarda notte seguente al giorno di qualche festa il canto notturno de' villani passeggeri. Infinità del passato che mi veniva in mente, ripensando ai Romani così caduti dopo tanto romore e ai tanti avvenimenti ora passati ch'io paragonava dolorosamente con quella profonda quiete e silenzio della notte, a farmi avvedere del quale giovava il risalto di quella voce o canto villanesco (98).

Ancora nello Zibaldone, a qualche anno di distanza rispetto al passo precedente, il poeta annotava:

Quello che altrove ho detto sugli effetti della luce, o degli oggetti visibili, in riguardo all'idea dell'infinito, si deve applicare parimente al suono, al canto, a tutto ciò che spetta all'udito. È piacevole per se stesso, cioè non per altro, se non per un'idea vaga ed indefinita che desta, un canto (il più spregevole) udito da lungi, o che paia lontano senza esserlo, o che si vada appoco appoco allontanando, e divenendo insensibile; o anche viceversa (ma meno), o che sia così lontano, in apparenza o in verità, che l'orecchio e l'idea quasi lo perda nella vastità degli spazi; un suono qua-

(94) Un esempio tra i molti: T. TAsso, Gerusalemme liberata XIX 71: "La fronte mesta / deh, per Dio! rasserena, e '1 duolo alleggia".

(95) Per avermi suggerito quest'ultimo passo sono grato a Luigi Blasucci, attento lettore del presente contributo nella sua originaria forma di tesi di laurea e fonte di puntualissime osservazioni.

(96) Leopardi, Poesie e Prose cit., II, p. 1188.

(97) LeOPARDI, Epistolario cit., pp. 653-4 (per cui cfr. BRUNI, Il canto della donna al telaio cit., p. 39). La lettera risale al 20 febbraio 1823.

(98) Zib. 50-51. 
lunque confuso, massime se ciò è per la lontananza; un canto udito in modo che non si veda il luogo da cui parte; un canto che risuoni per le volte di una stanza ec. dove voi non vi troviate però dentro; il canto degli agricoltori che nella campagna s'ode suonare per le valli, senza però vederli, e così il muggito degli armenti ec. Stando in casa, e udendo tali canti o suoni per la strada, massime di notte, si è più disposti a questi effetti, perché né l'udito né gli altri sensi non arrivano a determinare né circoscrivere la sensazione, e le sue concomitanze. È piacevole qualunque suono (anche vilissimo) che largamente e vastamente si diffonda, come in taluno dei detti casi, massime se non si vede l'oggetto da cui parte. [...] E tutte queste immagini in poesia ec. sono sempre bellissime, e tanto più quanto più negligentemente son messe, e toccando il soggetto, senza mostrar l'intenzione per cui ciò si fa, anzi mostrando d'ignorare l'effetto e le immagini che son per produrre, e di non toccarli se non per ispontanea, e necessaria congiuntura, e indole dell'argomento ec. V. in questo proposito Virg. Eneide 7. v.8. seqq. (99).

Sono numerosissimi i "magnetismi musicali" che percorrono le opere di Leopardi e che guidano i suoi passi fin dalla prima adolescenza al punto da dare vita ad una sorta di «diario "acustico"» (100); il canto, anche quello apparentemente più vile, colpisce, più di una visione, la sensibilità di Giacomo e brutalmente lo induce a riflettere sulla condizione di dolore sua e, in generale, del mondo. Acquista pregio ai suoi occhi la capacità dei poeti di inserire simili dettagli con noncuranza (negligentemente), di sfuggita, in modo che risultino quasi marginali; ma proprio da questi scaturisce un quadro d'insieme perfettamente delineato e il lettore, che sia attento non solo al mero aspetto narrativo, ne sarà affascinato. In questo senso va letto il canto di Simulo, un particolare che l'autore latino include, senza dargli troppo rilievo, tra le varie e triviali occupazioni del protagonista.

Sempre a proposito della vita contadina, non è sfuggito un legame tra il volgarizzamento e alcuni versi de La ginestra, in cui Leopardi immagina la fuga di un "meschino" (v. 249) e della sua famigliola per via dell'eruzione del Vesuvio; già al v. 252 compare "esplora [il corso / del temuto bollor]" - come Simulo esplora le tenebre dopo il risveglio, v. 7 - quindi l'uomo, ormai certo del pericolo,

desta i figliuoli,

desta la moglie in fretta, e via, con quanto

di lor cose rapir posson, fuggendo,

vede lontan l'usato

(99) Zib. 1927-30. Il rimando virgiliano è al canto di Circe, citato supra.

(100) M. OrCel, Il suono dell'Infinito. Saggi sulla poetica del primo Romanticismo italiano da Alfieri a Leopardi, trad. it. di P. Sodo, Napoli, Liguori, 1993, p. 99. Lo studioso mette in luce, soprattutto nell'idillio L'infinito (dove è proprio la percezione dello stormire del vento a scatenare il sovvenire), il ruolo assegnato dal poeta di Recanati ai suoni, e unisce i frammenti sparsi dello Zibaldone all'interno di una coerente teoria della musica quale forma sublime in cui la poesia in senso stretto deve confluire. Una traccia di questa spiccata sensibilità sonora è rinvenibile nell'inserimento, all'interno della Crestomazia dedicata alla prosa italiana, di un brano di Galileo intitolato eloquentemente La generazione dei suoni (cfr. G. LEOPARDI, Crestomazia italiana. La prosa, a cura di G. Bollati, Torino, Einaudi, 1968, pp. 122-4). Si veda inoltre BlasuCCI, Lo stormire del vento tra le piante cit., pp. 31-46. 
suo nido, e il picciol campo,

che gli fu dalla fame unico schermo (101).

Molte sono le spie di un possibile ricordo della Torta: anzitutto il verbo desta, più volte impiegato nel canto, è una variante per lo stampato "s'alza" (v. 6) del volgarizzamento, in una identica situazione di angoscia e timore, seppur per diversi motivi (102); in secondo luogo, ci riporta alla vicenda di Simulo la menzione di un campo annesso all'abitazione analogo al "breve campicel" (v. 4) della traduzione, ma soprattutto al "piccol/picciol campo" delle varianti (103) e al "piccol orto" del v. 85; determinante è l'evocazione dello spettro della fame dalla quale i prodotti agricoli tutelano entrambi i cultori. Era la fame la principale nemica di Simulo così come, ne La ginestra, lo è per il suo emulo leopardiano.

Infine, può essersi insinuata nella mente di Leopardi, per riemergere in seguito, un'ultima, più raffinata suggestione, che non compariva negli idilli greci: nel Moretum, Simulo ha come occupazione predominante il lavoro nei campi, mentre all'orto si dedica solo nei momenti in cui le condizioni meteorologiche gli impediscono attività quali l'aratura o la semina; i "dì di festa" (v. 88) ispirano prima di tutto una ambientazione (104): vi alludono perfino i titoli - La sera del dì di festa. Il giorno festivo ritorna ne Il sabato del villaggio, che evoca la vigilia della solennità: "ai dì della festa ella [la vecchierella] si ornava" (v. 12); "riede alla sua parca mensa, / fischiando, il zappatore, / e seco pensa al di del suo riposo" (vv. 2830 ) in cui svolgere quelle attività impraticabili nei giorni feriali; "[il legnaiuol] veglia / nella chiusa bottega alla lucerna, / e s'affretta, e s'adopra / di fornir l'opra anzi il chiarir dell'alba" (vv. 34-37), dove tornano "la lucerna" (v. 17) già di Simulo e un verbo, "chiarire", utilizzato intransitivamente dal poeta anche nella traduzione del Moretum (cfr. v. 20, riferito al fuoco). È tuttavia l'incipit de $I l$ sabato a contenere la traccia più palese della avvenuta fissazione, nella memoria del poeta, della traduzione latina:

La donzelletta vien dalla campagna,

in sul calar del sole,

col suo fascio dell'erba; e reca in mano

un mazzolin di rose e di viole,

onde, siccome suole,

ornare ella si appresta

dimani, al dì di festa, il petto e il crine (105).

(101) G. LEOPARDI, La ginestra o il fiore del deserto, vv. 260-5.

(102) Cfr. Stefani, "La Torta" di Giacomo Leopardi cit., p. 149 e F. D'InTino in LeOPARDI, Poeti latini e greci cit., p. 303.

(103) Cfr. Stefani, "La Torta" di Giacomo Leopardi cit., p. 149.

(104) "Un dì di Festa" compare anche ne Le Rimembranze, v. 36.

(105) G. LEOPARDI, Il sabato del villaggio, vv. 1-7. 
In questi pochi versi si nota una riproposizione, seppure scomposta in due momenti distinti, di un motivo della Torta:

Fasci d'erbe recando ei [Simulo] ne le None

da la compagna a la città venia,

e quindi a casa ritornar contento

scarco il capo solea, grave d'argento (106).

Ritornano i fasci d'erba, l'uso del verbo popolare-marchigiano "recare" (107), il cammino (di ritorno per la prima, di partenza per il secondo) dalla campagna alla città/villaggio, l'indicazione di una consuetudine (108). Nel complesso, si deduce che «il testo latino, assai descrittivo,» indusse Leopardi «a un ampliamento del linguaggio poetico in direzione del quotidiano; una lezione che fu messa a frutto nei grandi idilli di ambientazione paesana» (109). Si noti che il contento della traduzione recupera la precisa etimologia latina, indicante un pieno appagamento - come spesso nei Canti (110).

Risulta forse arduo pensare che, a distanza di anni, Leopardi abbia attinto, più o meno consapevolmente, a quel laboratorio poetico che fu la sua giovanile esperienza di traduttore. Tuttavia, come i

(106) G. LeOPARDI, La Torta, vv. 105-8. Sulle edizioni del Moretum presenti nella biblioteca di famiglia, Leopardi leggeva holerum (non umero) fasces portabat (cfr. Stefani, "La Torta" di Giacomo Leopardi cit., p. 143).

(107) Nella Lettera a P. Giordani del 30 maggio 1817, Leopardi scrive: «la nostra [dei Marchigiani] favella comune abbonda di frasi e motti e proverbi pretti toscani sì fattamente, che io mi meraviglio trovando negli scrittori una grandissima quantità di questi modi e idiotismi che ho imparati da fanciullo. E non mi fa meno stupore il sentire in bocca de' contadini e della plebe minuta parole che noi non usiamo nel favellare per fuggire l'affettazione, stimandole proprie dei soli Scrittori, come mentovato, ingombro, recare, ragionare ed altre molte [...]» (in LEOPARDI, Lettere cit., p. 78). Cfr. M. VERDUCCI, Lingua e stile: presenze e apporti dialettali negli scritti leopardiani, in Lingua e stile di Giacomo Leopardi. Atti dell'VIII Convegno internazionale di studi leopardiani - Recanati 30 settembre-5 ottobre 1991 (a cura di R. Garbuglia), Firenze, Olschki, 1994, p. 575.

(108) Cfr. S. Sconocchia, Tessuto linguistico e parola poetica nei Canti: l'incidenza dei classici, in Lingua e stile cit., p. 542; F. D'InTINo in LeOPard, Poeti latini e greci cit., pp. XXXVI e 309; D. ForNESI, Alcune considerazioni sul classicismo di Leopardi, in «Strumenti critici», n.s., XVI, 2001, p. 152; M. NATALE, Dagli Scherzi a Imitazione. Leopardi traduttore dei poeti: bibliografia 1955-2005, in «Lettere italiane», LVIII, 2006, p. 317; N. PRimo, Leopardi lettore e traduttore, Leonforte, Insula, 2008, p. 91.

(109) F. D'Intino in Leopard, Poeti latini e greci cit., p. 302. E cfr. N. Primo, Introduzione a G. SAVOCA-EADEM, Concordanza delle traduzioni poetiche di Giacomo Leopardi. Concordanza, lista di frequenza, indici, Firenze, Olschki, 2003, p. XXVII.

(110) Alla fine de La vita solitaria, vv. 106-7, il poeta sarà "assai contento / se core e lena a sospirar m'avanza”; Silvia è "assai contenta / di quel vago avvenir che in mente avevi" (A Silvia, vv. 11-12); anche in questo caso, l'aggettivo esprime la soddisfazione completa per una condizione certamente temporanea, ma, vista l'ingenuità dell'adolescente, vissuta appieno, senza la consapevolezza della sua precarietà. Ancora, "tu se' queta e contenta" è detto della greggia nel Canto notturno (v. 114) e la ginestra, nel canto omonimo, è "contenta dei deserti" (v. 7). 
Canti sono lì a indicare il ciclico ritorno di temi e motivi di particolare ispirazione dalle prime canzoni ai cosiddetti grandi idilli, così non si vede il motivo di negare l'altrettanto verosimile riemergere di sintagmi, e strutture linguistiche che con i primi vanno di pari passo. E questo infatti il tratto più sorprendente: se Leopardi ha la straordinaria capacità di creare nei suoi capolavori poetici situazioni e contesti diversi, ha anche quella di accompagnarli con le sue più riuscite espressioni linguistiche, che difficilmente sono riproposte con una funzione totalmente diversa. Il canto che allevia le fatiche è quello di un muratore, di un villano, di una fanciulla destinata a morire prematuramente; il campicello adibito a orto è di Simulo $e$ di un anonimo contadino del Vesuvio, allestito in entrambi i casi contro gli assalti della fame; i fasci d'erba sono recati ieri come oggi dalla campagna. Difficilmente un tema è affatto decontestualizzato e, trasposto di fiore in fiore, trascina con sé l'impalcatura linguistica su cui è emerso per la prima volta (111). Senza contare che la sensibilità dell'autore dei Canti è così antica che spesso le sue apparenti affinità con i classici non si possono ricondurre ad un modello precisamente individuato, ma scaturiscono da un gusto che sui classici è maturato (112) e che quei testi tende a far rivivere (113). Per questa ragione, se si pensa al Moretum o alla Torta come fonti, occorre tenere conto non solo dell' aspetto linguistico-espressivo, ma anche di quell'atmosfera generale che l'idillio suggerisce; come l'impianto eroico dell'Eneide si presta a riprese precise nelle canzoni, così l'ameno bozzetto latino bussa alla porta dell'autore degli Idilli riemergendo dai cassetti del suo scrittoio e della sua memoria letteraria (114). Il Moretum, così appassionatamente letto e tradotto in

(111) Ha scritto a questo proposito FORNESI, Alcune considerazioni sul classicismo di Leopardi cit., pp. 154-5: «Leopardi attribuisce un valore profondo a espressioni che sono quasi marche di determinate situazioni poetiche e vanno usate in un contesto a loro adatto».

(112) Cfr. E. Peruzzi, Studi leopardiani, I: La sera del di di festa, Firenze, Olschki, 1979, pp. 172-5 e 183: nel suo saggio, tra l'altro, lo studioso mostra come Leopardi sappia naturalmente descrivere «il risveglio della vita dopo il sonno della notte» alla maniera di Callimaco nella sua 'Exód $\eta$; sebbene Leopardi non potesse leggere quel passo specifico in quanto fu scoperto solo in seguito, egli, «poeta squisitamente, mirabilmente ellenico, per la precisione alessandrino» (SCONOCCHIA, La cultura classica cit., p. 316), fu capace di farsi a propria volta Callimaco. Identica «consonanza spirituale con gli antichi» Leopardi aveva già dimostrata nel canto di Simonide di All'Italia, dove aveva inserito dettagli pur attestati, ma da lui divinati, in quanto presenti in fonti non note, sul poeta lirico (cfr. E. PeruzzI, Studi leopardiani, II: Il canto di Simonide, Firenze, Olschki, 1987, pp. 65-69).

(113) Cfr. Sconocchia, La cultura classica cit., p. 287.

(114) Con procedimento quasi sillogistico ci si potrebbe appellare a L. BLASUCCI, Una fonte linguistica per $i$ "Canti": la traduzione del secondo libro dell' "Eneide" in Idem, Leopardi e $i$ segnali dell'infinito, Bologna, Il Mulino, 1985, pp. 9-30 per trovare almeno un precedente per questa consuetudine leopardiana di celare, anche a distanza di anni, nei Canti, riprese letterali non solo dei volgarizzamenti (Blasucci si 
gioventù, assurge al ruolo di primo idillio in quanto dona a Leopardi temi e motivi che, rielaborati alla luce di una ormai originale esperienza poetica, riemergeranno carsicamente nei Canti.

\section{JOHNNY L. BERTOLIO}

rifà a quello del secondo libro dell'Eneide), ma pure dell'originale latino. Su un piano differente, di recupero cioè diretto delle fonti classiche, greche in particolare, si articola l'analisi di G. LONARDI, Classicismo e utopia nella lirica leopardiana, Firenze, Olschki, 1969 e Idem, L'oro di Omero. L'Iliade”, Saffo: antichissimi di Leopardi, Venezia, Marsilio, 2005. 